\title{
qDSA: Small and Secure Digital Signatures with Curve-based Diffie-Hellman Key Pairs
}

\author{
Joost Renes ${ }^{1 \star}$ and Benjamin Smith $^{2}$ \\ 1 Digital Security Group, Radboud University, The Netherlands \\ j.renes@cs.ru.nl \\ 2 INRIA and Laboratoire d'Informatique de l'École polytechnique (LIX), \\ Université Paris-Saclay, France \\ smith@lix.polytechnique.fr
}

\begin{abstract}
DSA is a high-speed, high-security signature scheme that facilitates implementations with a very small memory footprint, a crucial requirement for embedded systems and IoT devices, and that uses the same public keys as modern Diffie-Hellman schemes based on Montgomery curves (such as Curve25519) or Kummer surfaces. qDSA resembles an adaptation of EdDSA to the world of Kummer varieties, which are quotients of algebraic groups by \pm 1 . Interestingly, qDSA does not require any full group operations or point recovery: all computations, including signature verification, occur on the quotient where there is no group law. We include details on four implementations of qDSA, using Montgomery and fast Kummer surface arithmetic on the 8-bit AVR ATmega and 32-bit ARM Cortex M0 platforms. We find that qDSA significantly outperforms state-of-the-art signature implementations in terms of stack usage and code size. We also include an efficient compression algorithm for points on fast Kummer surfaces, reducing them to the same size as compressed elliptic curve points for the same security level.

Keywords. Signatures, Kummer, Curve25519, Diffie-Hellman, elliptic curve, hyperelliptic curve.
\end{abstract}

\section{Introduction}

Modern asymmetric cryptography based on elliptic and hyperelliptic curves [29,31] achieves two important goals. The first is efficient key exchange using the Diffie-Hellman protocol [16], using the fact that the (Jacobian of the) curve carries the structure of an abelian group. But in fact, as Miller observed [31], we do not need the full group structure for Diffie-Hellman: the associated Kummer variety (the quotient by \pm 1 ) suffices, which permits more efficientlycomputable arithmetic [21,32]. Perhaps the most well-known example is Curve25519 [5], which offers fast scalar multiplications based on $x$-only arithmetic.

The second objective is efficient digital signatures, which are critical for authentication. There are several group-based signature schemes, the most important of which are ECDSA [1], Schnorr [40], and now EdDSA [8] signatures. In contrast to the Diffie-Hellman protocol, all of these signature schemes explicitly require the group structure of the (Jacobian of the) curve. An unfortunate side-effect of this is that users essentially need two public keys to support both curve-based protocols. Further, basic cryptographic libraries need to provide implementations for arithmetic on both the Jacobian and the Kummer variety, thus complicating and increasing the size of the trusted code base. For example, the NaCl library [9] uses Ed25519 [8] for signatures, and Curve25519 [5] for key exchange. This problem is worse for genus-2 hyperelliptic curves, where the Jacobian is significantly harder to use safely than its Kummer surface.

There have been several partial solutions to this problem. By observing that elements of the Kummer variety are elements of the Jacobian up to sign, one can build scalar multiplication

\footnotetext{
* This work has been supported by the Technology Foundation STW (project 13499 - TYPHOON \& ASPASIA), from the Dutch government.
} 
on the Jacobian based on the fast Kummer arithmetic [14,35]. This avoids the need for a separate scalar multiplication on the Jacobian, but does not avoid the need for its group law; it also introduces the need for projecting to and recovering from the Kummer. In any case, it does not solve the problem of having different public key types.

Another proposal is XEdDSA [36], which uses the public key on the Kummer variety to construct EdDSA signatures. In essence, it creates a key pair on the Jacobian by appending a sign bit to the public key on the Kummer variety, which can then be used for signatures. In [23] Hamburg shows that one can actually verify signatures using only the $x$-coordinates of points on an elliptic curve, which is applied in the recent STROBE framework [24]. We generalize this approach to allow Kummer varieties of curves of higher genera, and naturally adapt the scheme by only allowing challenges up to sign. This allows us to provide a proof of security, which has thus far not been attempted (in [23] Hamburg remarks that verifying up to sign does "probably not impact security at all"). Similar techniques have been applied for batch verification of ECDSA signatures [28], using the theory of summation polynomials [41].

In this paper we show that there is no intrinsic reason why Kummer varieties cannot be used for signatures. We present qDSA, a signature scheme relying only on Kummer arithmetic, and prove it secure in the random oracle model. It should not be surprising that the reduction in our proof is slightly weaker than the standard proof of security of Schnorr signatures [37], but not by more than we should expect. There is no difference between public keys for qDSA and Diffie-Hellman. After an abstract presentation in $\S 2$, we give a detailed description of ellipticcurve qDSA instances in $\S 3$. We then move on to genus-2 instances based on fast Kummer surfaces, which give better performance. The necessary arithmetic appears in $\S 4$, before $\S 5$ describes the new verification algorithm.

We also provide an efficient compression method for points on fast Kummer surfaces in $\S 6$, solving a long-standing open problem [6]. Our technique means that qDSA public keys for $g=2$ can be efficiently compressed to 32 bytes, and that qDSA signatures fit into 64 bytes; it also finally reduces the size of Kummer-based Diffie-Hellman public keys from 48 to 32 bytes.

Finally, we provide constant-time software implementations of genus- 1 and genus- 2 qDSA instances for the AVR ATmega and ARM Cortex M0 platforms. The performance of all four qDSA implementations, reported in $\S 7$, comfortably beats earlier implementations in terms of stack usage and code size.

Source code. We place all of the software described here into the public domain, to maximize the reusability of our results. The software is available at http://www.cs.ru.nl/ j jrenes/.

\section{The qDSA signature scheme}

In this section we define qDSA, the quotient Digital Signature Algorithm. We start by recalling the basics of Kummer varieties in $\S 2.1$ and defining key operations in $\S 2.2$. The rest of the section is dedicated to the definition of the qDSA signature scheme, which is presented in full in Algorithm 1, and its proof of security, which follows Pointcheval and Stern [37, 38]. qDSA closely resembles the Schnorr signature scheme [40], as it results from applying the Fiat-Shamir heuristic [19] to an altered Schnorr identification protocol, together with a few standard changes as in EdDSA [8]. We comment on some special properties of qDSA in $\S 2.5$.

Throughout, we work over finite fields $\mathbb{F}_{p}$ with $p>3$. Our low-level algorithms include costs

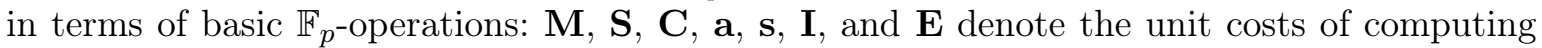


a single multiplication, squaring, multiplication by a small constant, addition, subtraction, inverse, and square root, respectively.

\subsection{The Kummer variety setting}

Let $\mathcal{C}$ be a (hyper)elliptic curve and $\mathcal{J}$ its Jacobian ${ }^{3}$. The Jacobian is a commutative algebraic group with group operation + , inverse - , and identity 0 . We assume $\mathcal{J}$ has a subgroup of large prime order $N$. The associated Kummer variety $\mathcal{K}$ is the quotient $\mathcal{K}=\mathcal{J} / \pm$. By definition, working with $\mathcal{K}$ corresponds to working on $\mathcal{J}$ up to sign. If $P$ is an element of $\mathcal{J}$, we denote its image in $\mathcal{K}$ by $\pm P$. In this paper we take $\log _{2} N \approx 256$, and consider two important cases.

Genus 1. Here $\mathcal{J}=\mathcal{C} / \mathbb{F}_{p}$ is an elliptic curve with $\log _{2} p \approx 256$, while $\mathcal{K}=\mathbb{P}^{1}$ is the $x$-line. We choose $\mathcal{C}$ to be Curve25519 [5], which is the topic of $\S 3$.

Genus 2. Here $\mathcal{J}$ is the Jacobian of a genus-2 curve $\mathcal{C} / \mathbb{F}_{p}$, where $\log _{2} p \approx 128$, and $\mathcal{K}$ is a Kummer surface. We use the Gaudry-Schost parameters [22] for our implementations. Kummer arithmetic, including some new constructions we need for signature verification and compression, is described in $\S 4-6$.

A point $\pm P$ in $\mathcal{K}\left(\mathbb{F}_{p}\right)$ is the image of a pair of points $\{P,-P\}$ on $\mathcal{J}$. It is important to note that $P$ and $-P$ are not necessarily in $\mathcal{J}\left(\mathbb{F}_{p}\right)$; if not, then they are conjugate points in $\mathcal{J}\left(\mathbb{F}_{p^{2}}\right)$, and correspond to points in $\mathcal{J}^{\prime}\left(\mathbb{F}_{p}\right)$, where $\mathcal{J}^{\prime}$ is the quadratic twist of $\mathcal{J}$. Both $\mathcal{J}$ and $\mathcal{J}^{\prime}$ always have the same Kummer variety; we return to this fact, and its implications for our scheme, in $\S 2.5$ below.

\subsection{Basic operations}

While a Kummer variety $\mathcal{K}$ has no group law, the operation

$$
\{ \pm P, \pm Q\} \mapsto\{ \pm(P+Q), \pm(P-Q)\}
$$

is well-defined. We can therefore define a pseudo-addition operation by

$$
\mathrm{xADD}:( \pm P, \pm Q, \pm(P-Q)) \mapsto \pm(P+Q) .
$$

The special case where $\pm(P-Q)= \pm 0$ is the pseudo-doubling xDBL $: \pm P \mapsto \pm[2] P$. In our applications we can often improve efficiency by combining two of these operations in a single function

$$
\text { xDBLADD : }( \pm P, \pm Q, \pm(P-Q)) \longmapsto( \pm[2] P, \pm(P+Q)) \text {. }
$$

For any integer $m$, the scalar multiplication $[m]$ on $\mathcal{J}$ induces the key cryptographic operation of pseudomultiplication on $\mathcal{K}$, defined by

$$
\text { Ladder }:(m, \pm P) \longmapsto \pm[m] P \text {. }
$$

As its name suggests, we compute Ladder using Montgomery's famous ladder algorithm [32], which is a uniform sequence of xDBLADDs and constant-time conditional swaps. ${ }^{4}$ This constanttime nature will be important for signing.

\footnotetext{
${ }^{3}$ In what follows, we could replace $\mathcal{J}$ by an arbitrary abelian group and all the proofs would be completely analogous. For simplicity we restrict to the cryptographically most interesting case of a Jacobian.

${ }^{4}$ In contemporary implementations such as $\mathrm{NaCl}$, the Ladder function is sometimes named crypto_scalarmult.
} 
Our signature verification requires a function Check on $\mathcal{K}^{3}$ defined by

$$
\text { Check : }( \pm P, \pm Q, \pm R) \longmapsto \begin{cases}\text { True } & \text { if } \pm R \in\{ \pm(P+Q), \pm(P-Q)\} \\ \text { False } & \text { otherwise }\end{cases}
$$

Since we are working with projective points, we need a way to uniquely represent them. Moreover, we want this representation to be as small as possible, to minimize communication overhead. For this purpose we define the functions

$$
\text { Compress }: \mathcal{K}\left(\mathbb{F}_{p}\right) \longrightarrow\{0,1\}^{256},
$$

writing $\overline{ \pm P}:=\operatorname{Compress}( \pm P)$, and

$$
\text { Decompress : }\{0,1\}^{256} \longrightarrow \mathcal{K}\left(\mathbb{F}_{p}\right) \cup\{\perp\}
$$

such that Decompress $(\overline{ \pm P})= \pm P$ for $\pm P$ in $\mathcal{K}\left(\mathbb{F}_{p}\right)$ and $\operatorname{Decompress}(X)=\perp$ for $X \in$ $\{0,1\}^{256} \backslash \operatorname{Im}$ (Compress).

For the remainder of this section we assume that Ladder, Check, Compress, and Decompress are defined. Their implementation depends on whether we are in the genus 1 or 2 setting; we return to this in later sections.

\subsection{The qID identification protocol}

Let $P$ be a generator of a prime order subgroup of $\mathcal{J}$, of order $N$, and let $\pm P$ be its image in $\mathcal{K}$. Let $\mathbb{Z}_{N}^{+}$denote the subset of $\mathbb{Z}_{N}$ with zero least significant bit (where we identify elements of $\mathbb{Z}_{N}$ with their representatives in $\left.[0, N-1]\right)$. Note that since $N$ is odd, $\operatorname{LSB}(-x)=1-\operatorname{LSB}(x)$ for all $x \in \mathbb{Z}_{N}^{*}$. The private key is an element $d \in \mathbb{Z}_{N}$. Let $Q=[d] P$ and let the public key be $\pm Q$. Now consider the following Schnorr-style identification protocol, which we call qID:

(1) The prover sets $r \leftarrow R \mathbb{Z}_{N}^{*}, \pm R \leftarrow \pm[r] P$ and sends $\pm R$ to the verifier;

(2) The verifier sets $c \leftarrow{ }_{R} \mathbb{Z}_{N}^{+}$and sends $c$ to the prover;

(3) The prover sets $s \leftarrow(r-c d) \bmod N$ and sends $s$ to the verifier;

(4) The verifier accepts if and only if $\pm R \in\{ \pm([s] P+[c] Q), \pm([s] P-[c] Q)\}$.

There are some important differences between qID and the basic Schnorr identification protocol in [40].

Scalar multiplications on $\mathcal{K}$. It is well-known that one can use $\mathcal{K}$ to perform the scalar multiplication $[14,35]$ within a Schnorr identification or signature scheme, but with this approach one must always lift back to an element of a group. In contrast, in our scheme this recovery step is not necessary.

Verification on $\mathcal{K}$. The original verification [40] requires checking that $R=[s] P+[c] Q$ for some $R,[s] P,[c] Q \in \mathcal{J}$. Working on $\mathcal{K}$, we only have these values up to sign (i. e. $\pm R$, $\pm[s] P$ and $\pm[c] Q)$, which is not enough to check that $R=[s] P+[c] Q$. Instead, we only verify that $\pm R= \pm([s] P \pm[c] Q)$.

Challenge from $\mathbb{Z}_{N}^{+}$. A Schnorr protocol using the weaker verification above would not satisfy the special soundness property: the transcripts $( \pm R, c, s)$ and $( \pm R,-c, s)$ are both valid, and do not allow us to extract a witness. Choosing $c$ from $\mathbb{Z}_{N}^{+}$instead of $\mathbb{Z}$ eliminates this possibility, and allows a security proof (this is the main difference with Hamburg's STROBE [24]). 
Proposition 1. The qID identification protocol is a sigma protocol.

Proof. We prove the required properties (see $[25, \S 6]$ ).

Completeness: If the protocol is followed, then $r=s+c d$, and therefore $[r] P=[s] P+[c] Q$ on $\mathcal{J}$. Mapping to $\mathcal{K}$, it follows that $\pm R= \pm([s] P+[c] Q)$.

Special soundness: Let $\left( \pm R, c_{0}, s_{0}\right)$ and $\left( \pm R, c_{1}, s_{1}\right)$ be two valid transcripts such that $c_{0} \neq c_{1}$. By verification, each $s_{i} \equiv \pm r \pm c_{i} d(\bmod N)$, so $s_{0} \pm s_{1} \equiv\left(c_{0} \pm c_{1}\right) d(\bmod N)$, where the signs are chosen to cancel $r$. Now $c_{0} \pm c_{1} \not \equiv 0(\bmod N)$ because $c_{0}$ and $c_{1}$ are both in $\mathbb{Z}_{N}^{+}$, so we can extract a witness $d \equiv\left(s_{0} \pm s_{1}\right)\left(c_{0} \pm c_{1}\right)^{-1}(\bmod N)$.

Honest-verifier zero-knowledge: A simulator $\mathcal{S}$ generates $c \leftarrow{ }_{R} \mathbb{Z}_{N}^{+}$and sets $s \leftarrow{ }_{R} \mathbb{Z}_{N}$ and $R \leftarrow[s] P+[c] Q .{ }^{5}$ If $R=\mathcal{O}$, it restarts. It outputs $( \pm R, c, s)$. As in [38, Lemma 5], we let

$$
\begin{aligned}
\delta & =\left\{( \pm R, c, s): c \in_{R} \mathbb{Z}_{N}^{+}, r \in_{R} \mathbb{Z}_{N}^{*}, \pm R= \pm[r] P, s=r-c d\right\}, \\
\delta^{\prime} & =\left\{( \pm R, c, s): c \in_{R} \mathbb{Z}_{N}^{+}, s \in_{R} \mathbb{Z}_{N}, R=[s] P+[c] Q, R \neq \mathcal{O}\right\}
\end{aligned}
$$

be the distributions of honest and simulated signatures, respectively. The elements of $\delta$ and $\delta^{\prime}$ are the same. First, consider $\delta$. There are exactly $N-1$ choices for $r$, and exactly $(N+1) / 2$ for $c$; all of them lead to distinct tuples. There are thus $\left(N^{2}-1\right) / 2$ possible tuples, all of which have probability $2 /\left(N^{2}-1\right)$ of occurring. Now consider $\delta^{\prime}$. Again, there are $(N+1) / 2$ choices for $c$. We have $N$ choices for $s$, exactly one of which leads to $R=\mathcal{O}$. Thus, given $c$, there are $N-1$ choices for $s$. We conclude that $\delta^{\prime}$ also contains $\left(N^{2}-1\right) / 2$ possible tuples, which all have probability $2 /\left(N^{2}-1\right)$ of occurring.

\subsection{Applying Fiat-Shamir}

Applying the Fiat-Shamir transform [19] to qID yields a signature scheme qSIG. We will need a hash function $\bar{H}:\{0,1\}^{*} \rightarrow \mathbb{Z}_{N}^{+}$, which we define by taking a hash function $H:\{0,1\}^{*} \rightarrow \mathbb{Z}_{N}$ and then setting $\bar{H}$ by

$$
\bar{H}(M) \longmapsto\left\{\begin{array}{ll}
H(M) & \text { if } \operatorname{LSB}(H(M))=0 \\
-H(M) & \text { if } \operatorname{LSB}(H(M))=1
\end{array} .\right.
$$

The qSIG signature scheme is defined as follows:

(1) To sign a message $M \in\{0,1\}^{*}$ with private key $d \in \mathbb{Z}_{N}$ and public key $\pm Q \in \mathcal{K}$, the prover sets $r \leftarrow R \mathbb{Z}_{N}^{*}, \pm R \leftarrow \pm[r] R, h \leftarrow \bar{H}( \pm R \| M)$, and $s \leftarrow(r-h d) \bmod N$, and sends $( \pm R \| s)$ to the verifier.

(2) To verify a signature $( \pm R \| s) \in \mathcal{K} \times \mathbb{Z}_{N}$ on a message $M \in\{0,1\}^{*}$ with public key $\pm Q \in \mathcal{K}$, the verifier sets $h \leftarrow \bar{H}( \pm R \| M), \pm \mathcal{T}_{0} \leftarrow \pm[s] P$, and $\pm \mathcal{T}_{1} \leftarrow \pm[h] Q$, and accepts if and only if $\pm R \in\left\{ \pm\left(\mathcal{T}_{0}+\mathcal{T}_{1}\right), \pm\left(\mathcal{T}_{0}-\mathcal{T}_{1}\right)\right\}$

Proposition 2 asserts that the security properties of qID carry over to qSIG.

Proposition 2. In the random oracle model, if an existential forgery of the qSIG signature scheme under an adaptive chosen message attack has non-negligible probability of success, then the DLP in $\mathcal{J}$ can be solved in polynomial time.

Proof. This is the standard proof of applying the Fiat-Shamir transform to a sigma protocol: see [37, Theorem 13] or [38, §3.2].

\footnotetext{
${ }^{5}$ As we only know $Q$ up to sign, we may need two attempts to construct $\mathcal{S}$.
} 


\subsection{The qDSA signature scheme}

Moving towards the real world, we slightly alter the qSIG protocol with some pragmatic choices, following Bernstein et al. [8]:

(1) We replace the randomness $r$ by the output of a pseudo-random function, which makes the signatures deterministic.

(2) We include the public key $\pm Q$ in the generation of the challenge, to prevent attackers from attacking multiple public keys at the same time.

(3) We compress and decompress points on $\mathcal{K}$ where necessary.

The resulting signature scheme, qDSA, is summarized in Algorithm 1.

Unified keys. Signatures are entirely computed and verified on $\mathcal{K}$, which is also the natural setting for Diffie-Hellman key exchange. We can therefore use identical key pairs for Diffie-Hellman and for qDSA signatures. This significantly simplifies the implementation of cryptographic libraries, as we no longer need arithmetic for the two distinct objects $\mathcal{J}$ and $\mathcal{K}$. Technically, there is no reason not to use a single key pair for both key exchange and signing; but one should be very careful in doing so, as using one key across multiple protocols could potentially lead to attacks. The primary interest of this aspect of qDSA is not necessarily in reducing the number of keys, but in unifying key formats and reducing the size of the trusted code base.

Security level. The security reduction to the discrete logarithm problem is almost identical to the case of Schnorr signatures [37]. Notably, the challenge space has about half the size $\left(\mathbb{Z}_{N}^{+}\right.$ versus $\mathbb{Z}_{N}$ ) while the proof of soundness computes either $s_{0}+s_{1}$ or $s_{0}-s_{1}$. This results in a slightly weaker reduction, as should be expected by moving from $\mathcal{J}$ to $\mathcal{K}$ and by weakening verification. By choosing $\log _{2} N \approx 256$ we obtain a scheme with about the same security level as state-of-the-art schemes (eg. EdDSA combined with Ed25519). This could be made more precise (cf. [38]), but we do not provide this analysis here.

Key and signature sizes. Public keys fit into 32 bytes in both the genus 1 and genus 2 settings. This is standard for Montgomery curves; for Kummer surfaces it requires a new compression technique, which we present in $\S 6$. In both cases $\log _{2} N<256$, which means that signatures $( \pm R \| s)$ fit in 64 bytes.

Twist security. Rational points on $\mathcal{K}$ correspond to pairs of points on either $\mathcal{J}$ or its quadratic twist. As opposed to Diffie-Hellman, in qDSA scalar multiplications with secret scalars are only performed on the public parameter $\pm P$, which is chosen as the image of large prime order element of $\mathcal{J}$. Therefore $\mathcal{J}$ is not technically required to have a secure twist, unlike in the modern Diffie-Hellman setting. But if $\mathcal{K}$ is also used for key exchange (which is the whole point!), then twist security is crucial. We therefore strongly recommend twist-secure parameters for qDSA implementations.

Hash function. The function $H$ can be any hash function with at least a $\log _{2} \sqrt{N}$-bit security level and at least $2 \log _{2} N$-bit output. Throughout this paper we take $H$ to be the extendable output function SHAKE128 [18] with fixed 512-bit output. This enables us to implicitly use $H$ as a function mapping into either $\mathbb{Z}_{N} \times\{0,1\}^{256}$ (eg. Line 3 of Algorithm 1 ), $\mathbb{Z}_{N}$ (eg. Line 8 of Algorithm 1), or $\mathbb{Z}_{N}^{+}$(eg. Line 11 of Algorithm 1, by combining it with a conditional negation) by appropriately reducing (part of) the output modulo $N$. 


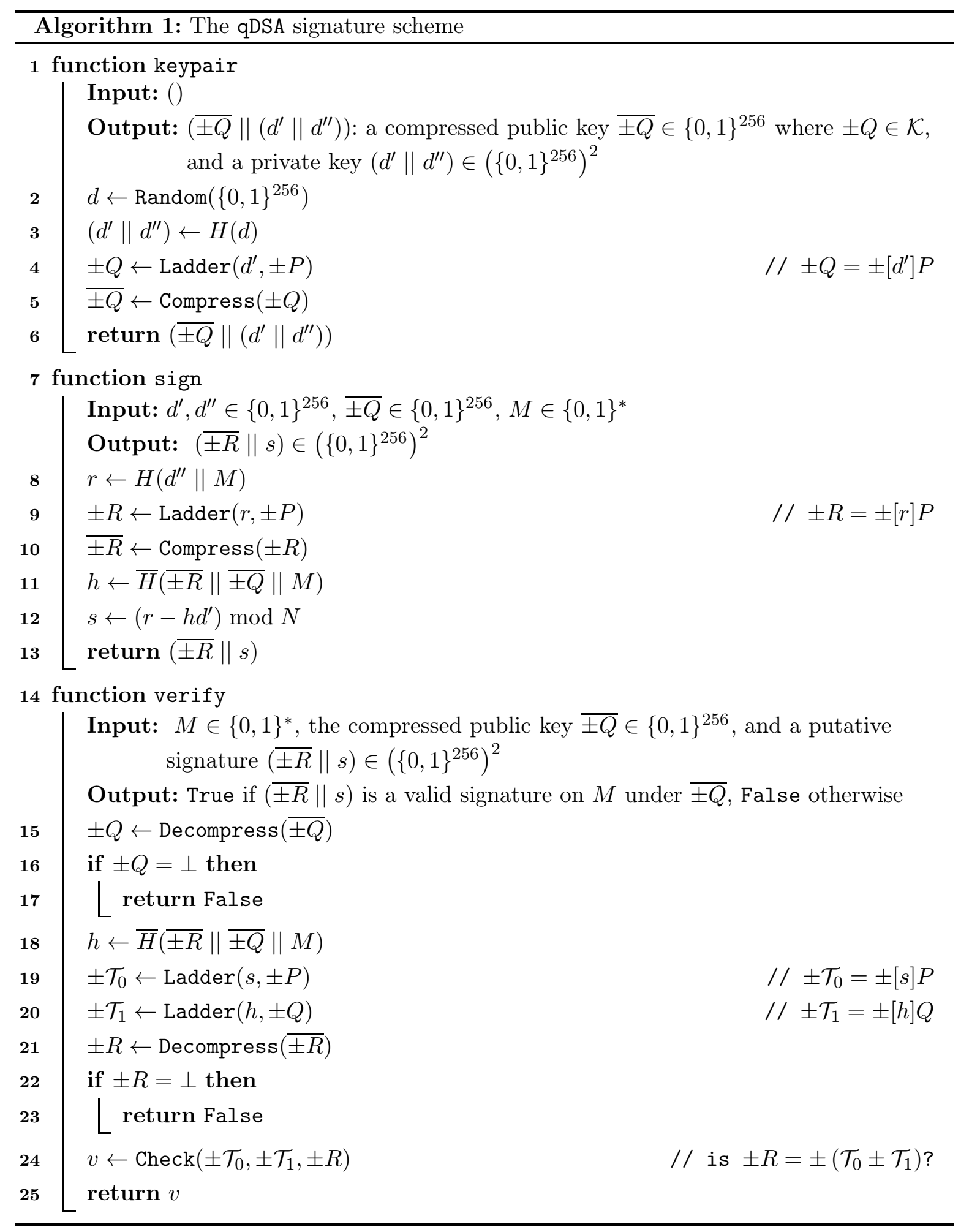


Signature compression. Schnorr mentions in [40] that signatures $(R \| s)$ may be compressed to $(H(R\|Q\| M) \| s)$, taking only the first 128 bits of the hash, thus reducing signature size from 64 to 48 bytes. This is possible because we can recompute $R$ from $P, Q, s$, and $H(R \|$ $Q \| M)$. However, on $\mathcal{K}$ we cannot recover $\pm R$ from $\pm P, \pm Q, s$, and $H( \pm R\| \pm Q\| M)$, so Schnorr's compression technique is not an option for us.

Batching. Proposals for batch signature verification typically rely on the group structure, verifying random linear combinations of points $[8,33]$. Since $\mathcal{K}$ has no group structure, these batching algorithms are not possible.

Scalar multiplication for verification. Instead of computing the full point $[s] P+[c] Q$ with a two-dimensional multiscalar multiplication operation, we have to compute $\pm[s] P$ and $\pm[c] Q$ separately. As a result we are unable to use standard tricks for speeding up two-dimensional scalar multiplications (eg. [20]), resulting in increased run-time. On the other hand, it has the benefit of relying on the already implemented Ladder function, mitigating the need for a separate algorithm, and is more memory-friendly. Our implementations show a significant decrease in stack usage, at the cost of a small loss of speed (see $\S 7$ ).

\section{Implementing qDSA with elliptic curves}

Our first concrete instantiation of qDSA uses the Kummer variety of an elliptic curve, which is just the $x$-line $\mathbb{P}^{1}$.

\subsection{Montgomery curves}

Consider the elliptic curve in Montgomery form

$$
E_{A B} / \mathbb{F}_{p}: B y^{2}=x\left(x^{2}+A x+1\right),
$$

where $A^{2} \neq 4$ and $B \neq 0$. The map $E_{A B} \rightarrow \mathcal{K}=\mathbb{P}^{1}$ defined by

$$
P=(X: Y: Z) \longmapsto \pm P= \begin{cases}(X: Z) & \text { if } Z \neq 0 \\ (1: 0) & \text { if } Z=0\end{cases}
$$

gives rise to efficient $x$-only arithmetic on $\mathbb{P}^{1}$ (see [32]). We use the Ladder specified in [17, Alg. 1]. Compression uses Bernstein's map

$$
\text { Compress : }(X: Z) \in \mathbb{P}^{1}\left(\mathbb{F}_{p}\right) \longmapsto X Z^{p-2} \in \mathbb{F}_{p},
$$

while decompression is the near-trivial

$$
\text { Decompress : } x \in \mathbb{F}_{p} \longmapsto(x: 1) \in \mathbb{P}^{1}\left(\mathbb{F}_{p}\right) \text {. }
$$

Note that Decompress never returns $\perp$, and that Decompress(Compress $((X: Z)))=(X: Z)$ whenever $Z \neq 0$ (however, the points $(0: 1)$ and $(1: 0)$ should never appear as public keys or signatures). 


\subsection{Signature verification}

It remains to define the Check operation for Montgomery curves. In the final step of verification we are given $\pm R, \pm P$, and $\pm Q$ in $\mathbb{P}^{1}$, and we need to check whether $\pm R \in\{ \pm(P+Q), \pm(P-Q)\}$. Proposition 3 reduces this to checking a quadratic relation in the coordinates of $\pm R, \pm P$, and $\pm Q$.

Proposition 3. Writing $\left(X^{P}: Z^{P}\right)= \pm P$ for $P$ in $E_{A B}$, etc.: If $P, Q$, and $R$ are points on $E_{A B}$, then $\pm R \in\{ \pm(P+Q), \pm(P-Q)\}$ if and only if

$$
B_{Z Z}\left(X^{R}\right)^{2}-2 B_{X Z} X^{R} Z^{R}+B_{X X}\left(Z^{R}\right)^{2}=0
$$

where

$$
\begin{aligned}
B_{X X} & =\left(X^{P} X^{Q}-Z^{P} Z^{Q}\right)^{2}, \\
B_{X Z} & =\left(X^{P} X^{Q}+Z^{P} Z^{Q}\right)\left(X^{P} Z^{Q}+Z^{P} X^{Q}\right)+2 A X^{P} Z^{P} X^{Q} Z^{Q}, \\
B_{Z Z} & =\left(X^{P} Z^{Q}-Z^{P} X^{Q}\right)^{2} .
\end{aligned}
$$

Proof. Let $S=\left(X^{S}: Z^{S}\right)= \pm(P+Q)$ and $D=\left(X^{D}: Z^{D}\right)= \pm(P-Q)$. If we temporarily assume $\pm 0 \neq \pm P \neq \pm Q \neq \pm 0$ and put $x_{P}=X^{P} / Z^{P}$, etc., then the group law on $E_{A B}$ gives us $x_{S} x_{D}=\left(x_{P} x_{Q}-1\right)^{2} /\left(x_{P}-x_{Q}\right)^{2}$ and $x_{S}+x_{D}=2\left(\left(x_{P} x_{Q}+1\right)\left(x_{P}+x_{Q}\right)+2 A x_{P} x_{Q}\right)$. Homogenizing, we obtain

$$
\left(X^{S} X^{D}: X^{S} Z^{D}+Z^{S} X^{D}: Z^{S} Z^{D}\right)=\left(\lambda B_{X X}: \lambda 2 B_{X Z}: \lambda B_{Z Z}\right) .
$$

One readily verifies that Equation (6) still holds even when the temporary assumption does not (that is, when $\pm P= \pm Q$ or $\pm P= \pm 0$ or $\pm Q= \pm 0$ ). Having degree 2, the homogeneous polynomial $B_{Z Z} X^{2}-B_{X Z} X Z+B_{X X} Z^{2}$ cuts out two points in $\mathbb{P}^{1}$ (which may coincide); by Equation (6), they are $\pm(P+Q)$ and $\pm(P-Q)$, so if $\left(X^{R}: Z^{R}\right)$ satisfies Equation (2) then it must be one of them.

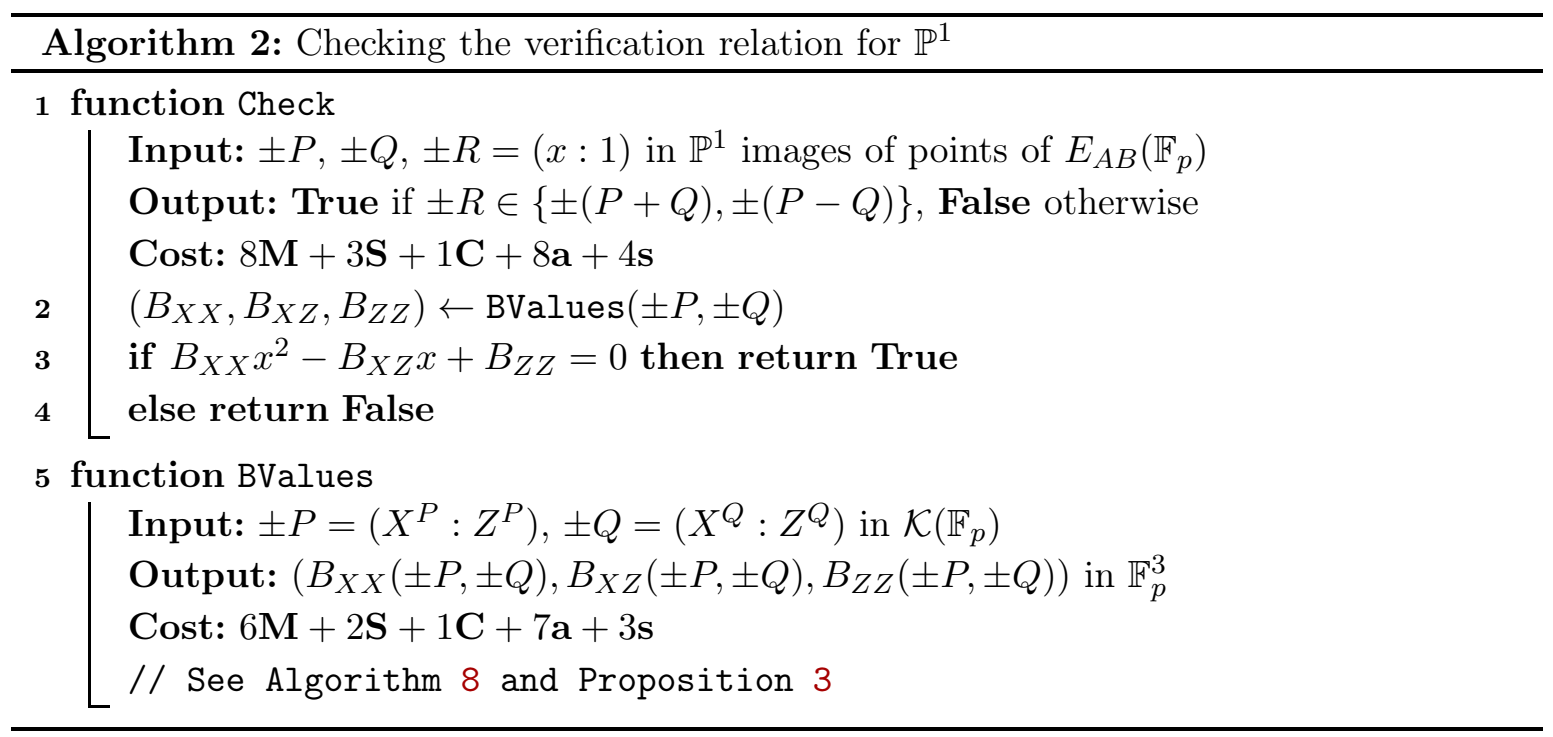




\subsection{Using cryptographic parameters}

We use the elliptic curve $E / \mathbb{F}_{p}: y^{2}=x^{3}+486662 x^{2}+x$ where $p=2^{255}-19$, which is commonly referred to as Curve25519 [5]. Let $P \in E\left(\mathbb{F}_{p}\right)$ be such that $\pm P=(9: 1)$. Then $P$ has order $8 N$, where

$$
N=2^{252}+27742317777372353535851937790883648493
$$

is prime. The xDBLADD operation requires us to store $(A+2) / 4=121666$, and we implement optimized multiplication by this constant. In [5, §3] Bernstein sets and clears some bits of the private key, also referred to as "clamping". This is not necessary in qDSA, but we do it anyway in keypair for compatibility.

\section{Implementing qDSA with Kummer surfaces}

A number of cryptographic protocols that have been successfully implemented with Montgomery curves have seen substantial practical improvements when the curves are replaced with Kummer surfaces. From a general point of view, a Kummer surface is the quotient of some genus-2 Jacobian $\mathcal{J}$ by \pm 1 ; geometrically it is a surface in $\mathbb{P}^{3}$ with sixteen point singularities, called nodes, which are the images in $\mathcal{K}$ of the 2 -torsion points of $\mathcal{J}$ (since these are precisely the points fixed by -1 ). From a cryptographic point of view, a Kummer surface is just a 2-dimensional analogue of the $x$-coordinate used in Montgomery curve arithmetic.

The algorithmic and software aspects of efficient Kummer surface arithmetic have already been covered in great detail elsewhere (see eg. [21], [7], and [39]). Indeed, the Kummer scalar multiplication algorithms and software that we use in our signature implementation are identical to those described in [39], and use the cryptographic parameters proposed by Gaudry and Schost [22].

This work includes two entirely new Kummer algorithms that are essential for our signature scheme: verification relation testing (Check, Algorithm 3) and compression/decompression (Compress and Decompress, Algorithms 4 and 5). Both of these new techniques require a fair amount of technical development, which we begin in this section by recalling the basic Kummer equation and constants, and deconstructing the pseudo-doubling operation into a sequence of surfaces and maps that will play important roles later. Once the scene has been set, we will describe our signature verification algorithm in $\S 5$ and our point compression scheme in $\S 6$. The reader primarily interested in the resulting performance improvements may wish to skip directly to $\S 7$ on first reading.

The Check, Compress, and Decompress algorithms defined below require the following subroutines:

- Mul4 implements a 4 -way parallel multiplication. It takes a pair of vectors $\left(x_{1}, x_{2}, x_{3}, x_{4}\right)$ and $\left(y_{1}, y_{2}, y_{3}, y_{4}\right)$ in $\mathbb{F}_{p}^{4}$, and returns $\left(x_{1} y_{1}, x_{2} y_{2}, x_{3} y_{3}, x_{4} y_{4}\right)$.

- Sqr4 implements a 4 -way parallel squaring. Given a vector $\left(x_{1}, x_{2}, x_{3}, x_{4}\right)$ in $\mathbb{F}_{p}^{4}$, it returns $\left(x_{1}^{2}, x_{2}^{2}, x_{3}^{2}, x_{4}^{2}\right)$.

- Had implements a Hadamard transform. Given a vector $\left(x_{1}, x_{2}, x_{3}, x_{4}\right)$ in $\mathbb{F}_{p}^{4}$, it returns $\left(x_{1}+x_{2}+x_{3}+x_{4}, x_{1}+x_{2}-x_{3}-x_{4}, x_{1}-x_{2}+x_{3}-x_{4}, x_{1}-x_{2}-x_{3}+x_{4}\right)$.

- Dot computes the sum of a 4-way multiplication. Given a pair of vectors $\left(x_{1}, x_{2}, x_{3}, x_{4}\right)$ and $\left(y_{1}, y_{2}, y_{3}, y_{4}\right)$ in $\mathbb{F}_{p}^{4}$, it returns $x_{1} y_{1}+x_{2} y_{2}+x_{3} y_{3}+x_{4} y_{4}$. 


\subsection{Constants}

Our Kummer surfaces are defined by four fundamental constants $\alpha_{1}, \alpha_{2}, \alpha_{3}, \alpha_{4}$ and four dual constants $\widehat{\alpha}_{1}, \widehat{\alpha}_{2}, \widehat{\alpha}_{3}$, and $\widehat{\alpha}_{4}$, which are related by

$$
\begin{aligned}
& 2 \widehat{\alpha}_{1}^{2}=\alpha_{1}^{2}+\alpha_{2}^{2}+\alpha_{3}^{2}+\alpha_{4}^{2}, \\
& 2 \widehat{\alpha}_{2}^{2}=\alpha_{1}^{2}+\alpha_{2}^{2}-\alpha_{3}^{2}-\alpha_{4}^{2}, \\
& 2 \widehat{\alpha}_{3}^{2}=\alpha_{1}^{2}-\alpha_{2}^{2}+\alpha_{3}^{2}-\alpha_{4}^{2}, \\
& 2 \widehat{\alpha}_{4}^{2}=\alpha_{1}^{2}-\alpha_{2}^{2}-\alpha_{3}^{2}+\alpha_{4}^{2} .
\end{aligned}
$$

We require all of the $\alpha_{i}$ and $\widehat{\alpha}_{i}$ to be nonzero. The fundamental constants determine the dual constants up to sign, and vice versa. These relations remain true when we exchange the $\alpha_{i}$ with the $\widehat{\alpha}_{i}$; we call this "swapping $x$ with $\widehat{x}$ " operation "dualizing". To make the symmetry in what follows clear, we define

$$
\begin{array}{lll}
\mu_{1}:=\alpha_{1}^{2}, & \epsilon_{1}:=\mu_{2} \mu_{3} \mu_{4}, & \kappa_{1}:=\epsilon_{1}+\epsilon_{2}+\epsilon_{3}+\epsilon_{4}, \\
\mu_{2}:=\alpha_{2}^{2}, & \epsilon_{2}:=\mu_{1} \mu_{3} \mu_{4}, & \kappa_{2}:=\epsilon_{1}+\epsilon_{2}-\epsilon_{3}-\epsilon_{4}, \\
\mu_{3}:=\alpha_{3}^{2}, & \epsilon_{3}:=\mu_{1} \mu_{2} \mu_{4}, & \kappa_{3}:=\epsilon_{1}-\epsilon_{2}+\epsilon_{3}-\epsilon_{4}, \\
\mu_{4}:=\alpha_{4}^{2}, & \epsilon_{4}:=\mu_{1} \mu_{2} \mu_{3}, & \kappa_{4}:=\epsilon_{1}-\epsilon_{2}-\epsilon_{3}+\epsilon_{4},
\end{array}
$$

along with their respective duals $\widehat{\mu}_{i}, \widehat{\epsilon}_{i}$, and $\widehat{\kappa}_{i}$. Note that

$$
\left(\epsilon_{1}: \epsilon_{2}: \epsilon_{3}: \epsilon_{4}\right)=\left(1 / \mu_{1}: 1 / \mu_{2}: 1 / \mu_{3}: 1 / \mu_{4}\right)
$$

and $\mu_{i} \mu_{j}-\mu_{k} \mu_{l}=\widehat{\mu}_{i} \widehat{\mu}_{j}-\widehat{\mu}_{k} \widehat{\mu}_{l}$ for $\{i, j, k, l\}=\{1,2,3,4\}$. There are many clashing notational conventions for theta constants in the cryptographic Kummer literature; Table 1 provides a dictionary for converting between them.

Our applications use only the squared constants $\mu_{i}$ and $\widehat{\mu}_{i}$, so only they need be in $\mathbb{F}_{p}$. In practice we want them to be as "small" as possible, both to reduce the cost of multiplying by them and to reduce the cost of storing them. In fact, it follows from their definition that it is much easier to find simultaneously small $\mu_{i}$ and $\widehat{\mu}_{i}$ than it is to find simultaneously small $\alpha_{i}$ and $\widehat{\alpha}_{i}$ (or a mixture of the two); this is ultimately why we prefer the squared surface for scalar multiplication. We note that if the $\mu_{i}$ are very small, then the $\epsilon_{i}$ and $\kappa_{i}$ are also small, and the same goes for their duals. While we will never actually compute with the unsquared constants, we need them to explain what is happening in the background below.

Finally, the Kummer surface equations involve some derived constants

$$
\begin{gathered}
E:=\frac{16 \alpha_{1} \alpha_{2} \alpha_{3} \alpha_{4} \widehat{\mu}_{1} \widehat{\mu}_{2} \widehat{\mu}_{3} \widehat{\mu}_{4}}{\left(\mu_{1} \mu_{4}-\mu_{2} \mu_{3}\right)\left(\mu_{1} \mu_{3}-\mu_{2} \mu_{4}\right)\left(\mu_{1} \mu_{2}-\mu_{3} \mu_{4}\right)}, \\
F:=2 \frac{\mu_{1} \mu_{4}+\mu_{2} \mu_{3}}{\mu_{1} \mu_{4}-\mu_{2} \mu_{3}}, \quad G:=2 \frac{\mu_{1} \mu_{3}+\mu_{2} \mu_{4}}{\mu_{1} \mu_{3}-\mu_{2} \mu_{4}}, \quad H:=2 \frac{\mu_{1} \mu_{2}+\mu_{3} \mu_{4}}{\mu_{1} \mu_{2}-\mu_{3} \mu_{4}},
\end{gathered}
$$

and their duals $\widehat{E}, \widehat{F}, \widehat{G}, \widehat{H}$. We observe that $E^{2}=F^{2}+G^{2}+H^{2}+F G H-4$ and $\widehat{E}^{2}=$ $\widehat{F}^{2}+\widehat{G}^{2}+\widehat{H}^{2}+\widehat{F} \widehat{G} \widehat{H}-4$. 


\begin{tabular}{c|c|c} 
Source & Fundamental constants & Dual constants \\
\hline [21] and [7] & $(a: b: c: d)=\left(\alpha_{1}: \alpha_{2}: \alpha_{3}: \alpha_{4}\right)$ & $(A: B: C: D)=\left(\widehat{\alpha}_{1}: \widehat{\alpha}_{2}: \widehat{\alpha}_{3}: \widehat{\alpha}_{4}\right)$ \\
{$[11]$} & $(a: b: c: d)=\left(\alpha_{1}: \alpha_{2}: \alpha_{3}: \alpha_{4}\right)$ & $(A: B: C: D)=\left(\widehat{\mu}_{1}: \widehat{\mu}_{2}: \widehat{\mu}_{3}: \widehat{\mu}_{4}\right)$ \\
{$[39]$} & $(a: b: c: d)=\left(\mu_{1}: \mu_{2}: \mu_{3}: \mu_{4}\right)$ & $(A: B: C: D)=\left(\widehat{\mu}_{1}: \widehat{\mu}_{2}: \widehat{\mu}_{3}: \widehat{\mu}_{4}\right)$ \\
{$[15]$} & $(\alpha: \beta: \gamma: \delta)=\left(\mu_{1}: \mu_{2}: \mu_{3}: \mu_{4}\right)$ & $(A: B: C: D)=\left(\widehat{\mu}_{1}: \widehat{\mu}_{2}: \widehat{\mu}_{3}: \widehat{\mu}_{4}\right)$
\end{tabular}

Table 1. Relations between our theta constants and others in selected related work

\subsection{Fast Kummer surfaces}

We compute all of the pseudoscalar multiplications in qDSA on the so-called squared Kummer surface

$$
\mathcal{K}^{\mathrm{Sqr}}: 4 E^{2} \cdot X_{1} X_{2} X_{3} X_{4}=\left(\begin{array}{c}
X_{1}^{2}+X_{2}^{2}+X_{3}^{2}+X_{4}^{2}-F\left(X_{1} X_{4}+X_{2} X_{3}\right) \\
-G\left(X_{1} X_{3}+X_{2} X_{4}\right)-H\left(X_{1} X_{2}+X_{3} X_{4}\right)
\end{array}\right)^{2},
$$

which was proposed for factorization algorithms by the Chudnovskys [13], then later for DiffieHellman by Bernstein [6]. Since $E$ only appears as a square, $\mathcal{K}^{\text {Sqr }}$ is defined over $\mathbb{F}_{p}$. The zero point on $\mathcal{K}^{\mathrm{Sqr}}$ is $\pm 0=\left(\mu_{1}: \mu_{2}: \mu_{3}: \mu_{4}\right)$. In our implementations we used the xDBLADD and Montgomery ladder exactly as they were presented in [39, Algorithms 6-7] (see also Algorithm 9). The pseudo-doubling xDBL on $\mathcal{K}^{\text {Sqr }}$ is

$$
\pm P=\left(X_{1}^{P}: X_{2}^{P}: X_{3}^{P}: X_{4}^{P}\right) \longmapsto\left(X_{1}^{[2] P}: X_{2}^{[2] P}: X_{3}^{[2] P}: X_{4}^{[2] P}\right)= \pm[2] P
$$

where

$$
\begin{array}{ll}
X_{1}^{[2] P}=\epsilon_{1}\left(U_{1}+U_{2}+U_{3}+U_{4}\right)^{2}, & U_{1}=\widehat{\epsilon}_{1}\left(X_{1}^{P}+X_{2}^{P}+X_{3}^{P}+X_{4}^{P}\right)^{2}, \\
X_{2}^{[2] P}=\epsilon_{2}\left(U_{1}+U_{2}-U_{3}-U_{4}\right)^{2}, & U_{2}=\widehat{\epsilon}_{2}\left(X_{1}^{P}+X_{2}^{P}-X_{3}^{P}-X_{4}^{P}\right)^{2}, \\
X_{3}^{[2] P}=\epsilon_{3}\left(U_{1}-U_{2}+U_{3}-U_{4}\right)^{2}, & U_{3}=\widehat{\epsilon}_{3}\left(X_{1}^{P}-X_{2}^{P}+X_{3}^{P}-X_{4}^{P}\right)^{2}, \\
X_{4}^{[2] P}=\epsilon_{4}\left(U_{1}-U_{2}-U_{3}+U_{4}\right)^{2}, & U_{4}=\widehat{\epsilon}_{4}\left(X_{1}^{P}-X_{2}^{P}-X_{3}^{P}+X_{4}^{P}\right)^{2},
\end{array}
$$

for $\pm P$ with all $X_{i}^{P} \neq 0$; more complicated formulæ exist for other $\pm P$ (cf. $\S 5.1$ ).

\subsection{Deconstructing pseudo-doubling}

Figure 1 deconstructs the pseudo-doubling on $\mathcal{K}^{\text {Sqr }}$ from $\S 4.2$ into a cycle of atomic maps between different Kummer surfaces, which form a sort of hexagon. Starting at any one of the Kummers and doing a complete cycle of these maps carries out pseudo-doubling on that Kummer. Doing a half-cycle from a given Kummer around to its dual computes a $(2,2)$-isogeny splitting pseudo-doubling.

Six different Kummer surfaces may seem like a lot to keep track of - even if there are really only three, together with their duals. However, the new surfaces are important, because they are crucial in deriving our Check routine (of course, once the algorithm has been written down, the reader is free to forget about the existence of these other surfaces).

The cycle actually begins one step before $\mathcal{K}^{\mathrm{Sqr}}$, with the canonical surface

$$
\mathcal{K}^{\text {Can }}: 2 E \cdot T_{1} T_{2} T_{3} T_{4}=\begin{aligned}
& T_{1}^{4}+T_{2}^{4}+T_{3}^{4}+T_{4}^{4}-F\left(T_{1}^{2} T_{4}^{2}+T_{2}^{2} T_{3}^{2}\right) \\
& -G\left(T_{1}^{2} T_{3}^{2}+T_{2}^{2} T_{4}^{2}\right)-H\left(T_{1}^{2} T_{2}^{2}+T_{3}^{2} T_{4}^{2}\right) .
\end{aligned}
$$




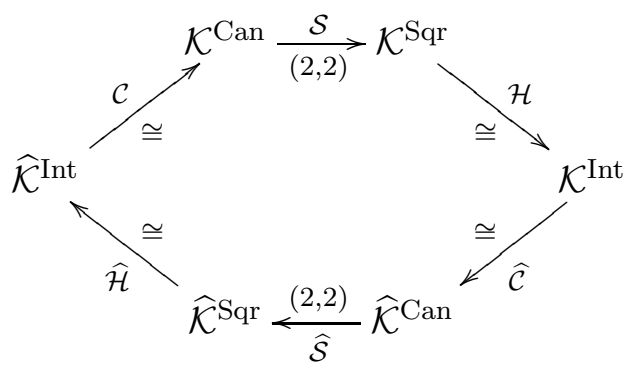

Fig. 1. Decomposition of pseudo-doubling on fast Kummer surfaces into a cycle of morphisms. Here, $\mathcal{K}^{\text {Sqr }}$ is the "squared" surface we mostly compute with; $\mathcal{K}^{\text {Can }}$ is the related "canonical" surface; and $\mathcal{K}^{\text {Int }}$ is a new "intermediate" surface which we use in signature verification. (The surfaces $\widehat{\mathcal{K}}^{\text {Sqr }}, \widehat{\mathcal{K}}^{\text {Can }}$, and $\widehat{\mathcal{K}}^{\text {Int }}$ are their duals.)

This was the model proposed for cryptographic applications by Gaudry in [21]; we call it "canonical" because it is the model arising from a canonical basis of theta functions of level $(2,2)$.

Now we can begin our tour around the hexagon, moving from $\mathcal{K}^{\mathrm{Can}}$ to $\mathcal{K}^{\mathrm{Sqr}}$ via the squaring map

$$
\mathcal{S}:\left(T_{1}: T_{2}: T_{3}: T_{4}\right) \longmapsto\left(X_{1}: X_{2}: X_{3}: X_{4}\right)=\left(T_{1}^{2}: T_{2}^{2}: T_{3}^{2}: T_{4}^{3}\right) \text {, }
$$

which corresponds to a $(2,2)$-isogeny of Jacobians. Moving on from $\mathcal{K}^{\text {Sqr }}$, the Hadamard transform isomorphism

$$
\mathcal{H}:\left(X_{1}: X_{2}: X_{3}: X_{4}\right) \longmapsto\left(Y_{1}: Y_{2}: Y_{3}: Y_{4}\right)=\left(\begin{array}{c}
X_{1}+X_{2}+X_{3}+X_{4} \\
: X_{1}+X_{2}-X_{3}-X_{4} \\
: X_{1}-X_{2}+X_{3}-X_{4} \\
: X_{1}-X_{2}-X_{3}+X_{4}
\end{array}\right)
$$

takes us into a third kind of Kummer, which we call the intermediate surface:

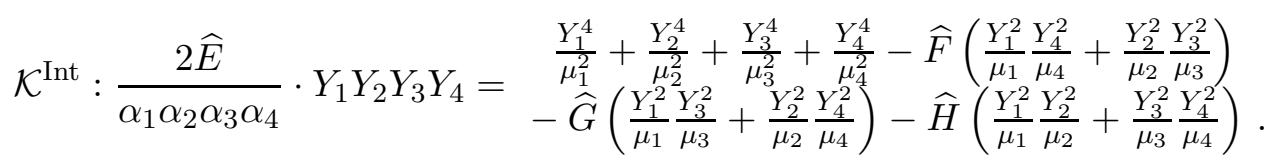

We will use $\mathcal{K}^{\text {Int }}$ for signature verification. Now the dual scaling isomorphism

$$
\widehat{\mathcal{C}}:\left(Y_{1}: Y_{2}: Y_{3}: Y_{4}\right) \longmapsto\left(\widehat{T}_{1}: \widehat{T}_{2}: \widehat{T}_{3}: \widehat{T}_{4}\right)=\left(Y_{1} / \widehat{\alpha}_{1}: Y_{2} / \widehat{\alpha}_{2}: Y_{3} / \widehat{\alpha}_{3}: Y_{4} / \widehat{\alpha}_{4}\right)
$$

takes us into the dual canonical surface

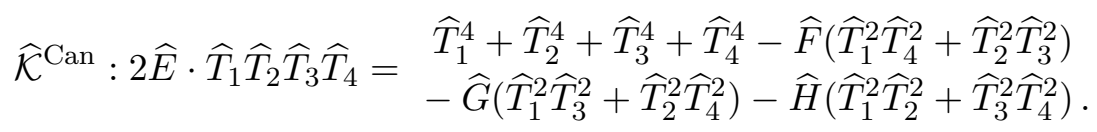

We are now halfway around the hexagon; the return journey is simply the dual of the outbound trip. The dual squaring map

$$
\widehat{\mathcal{S}}:\left(\widehat{T}_{1}: \widehat{T}_{2}: \widehat{T}_{3}: \widehat{T}_{4}\right) \longmapsto\left(\widehat{X}_{1}: \widehat{X}_{2}: \widehat{X}_{3}: \widehat{X}_{4}\right)=\left(\widehat{T}_{1}^{2}: \widehat{T}_{2}^{2}: \widehat{T}_{3}^{2}: \widehat{T}_{4}^{3}\right),
$$


another (2,2)-isogeny, carries us into the dual squared surface

$$
\widehat{\mathcal{K}}^{\mathrm{Sqr}}: 4 \widehat{E}^{2} \cdot \widehat{X}_{1} \widehat{X}_{2} \widehat{X}_{3} \widehat{X}_{4}=\left(\begin{array}{c}
\widehat{X}_{1}^{2}+\widehat{X}_{2}^{2}+\widehat{X}_{3}^{2}+\widehat{X}_{4}^{2}-\widehat{F}\left(\widehat{X}_{1} \widehat{X}_{4}+\widehat{X}_{2} \widehat{X}_{3}\right) \\
-\widehat{G}\left(\widehat{X}_{1} \widehat{X}_{3}+\widehat{X}_{2} \widehat{X}_{4}\right)-\widehat{H}\left(\widehat{X}_{1} \widehat{X}_{2}+\widehat{X}_{3} \widehat{X}_{4}\right)
\end{array}\right)^{2},
$$

before the dual Hadamard transform

$$
\widehat{\mathcal{H}}:\left(\widehat{X}_{1}: \widehat{X}_{2}: \widehat{X}_{3}: \widehat{X}_{4}\right) \longmapsto\left(\widehat{Y}_{1}: \widehat{Y}_{2}: \widehat{Y}_{3}: \widehat{Y}_{4}\right)=\left(\begin{array}{c}
\widehat{X}_{1}+\widehat{X}_{2}+\widehat{X}_{3}+\widehat{X}_{4} \\
: \widehat{X}_{1}+\widehat{X}_{2}-\widehat{X}_{3}-\widehat{X}_{4} \\
: \widehat{X}_{1}-\widehat{X}_{2}+\widehat{X}_{3}-\widehat{X}_{4} \\
: \widehat{X}_{1}-\widehat{X}_{2}-\widehat{X}_{3}+\widehat{X}_{4}
\end{array}\right)
$$

takes us into the dual intermediate surface

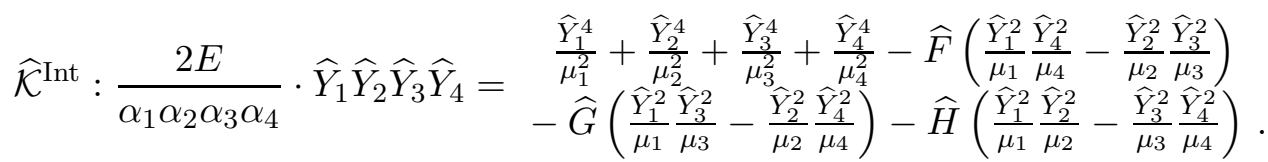

A final scaling isomorphism

$$
\mathcal{C}:\left(\widehat{Y}_{1}: \widehat{Y}_{2}: \widehat{Y}_{3}: \widehat{Y}_{4}\right) \longmapsto\left(T_{1}: T_{2}: T_{3}: T_{4}\right)=\left(\widehat{Y}_{1} / \alpha_{1}: \widehat{Y}_{2} / \alpha_{2}: \widehat{Y}_{3} / \alpha_{3}: \widehat{Y}_{4} / \alpha_{4}\right)
$$

takes us from $\widehat{\mathcal{K}}^{\text {Int }}$ back to $\mathcal{K}^{\text {Can }}$, where we started.

The canonical surfaces $\mathcal{K}^{\text {Can }}$ resp. $\widehat{\mathcal{K}}^{\text {Can }}$ are only defined over $\mathbb{F}_{p}\left(\alpha_{1} \alpha_{2} \alpha_{3} \alpha_{4}\right)$ resp. $\mathbb{F}_{p}\left(\widehat{\alpha}_{1} \widehat{\alpha}_{2} \widehat{\alpha}_{3} \widehat{\alpha}_{4}\right)$, while the scaling isomorphisms $\widehat{\mathcal{C}}$ resp. $\mathcal{C}$ are defined over $\mathbb{F}_{p}\left(\widehat{\alpha}_{1}, \widehat{\alpha}_{2}, \widehat{\alpha}_{3}, \widehat{\alpha}_{4}\right)$ resp. $\mathbb{F}_{p}\left(\alpha_{1}, \alpha_{2}, \alpha_{3}, \alpha_{4}\right)$. Everything else is defined over $\mathbb{F}_{p}$.

We confirm that one cycle around the hexagon, starting and ending on $\mathcal{K}^{\mathrm{Sqr}}$, computes the pseudo-doubling of Equations (7), (8), (9), and (10). Similarly, one cycle around the hexagon starting and ending on $\mathcal{K}^{\mathrm{Can}}$ computes Gaudry's pseudo-doubling from [21, §3.2].

\section{Signature verification on Kummer surfaces}

To verify signatures in the Kummer surface implementation, we need to supply a Check algorithm which, given $\pm P, \pm Q$, and $\pm R$ on $\mathcal{K}^{\mathrm{Sqr}}$, decides whether $\pm R \in\{ \pm(P+Q), \pm(P-$ $Q)\}$. For the elliptic version of qDSA described in $\S 3$, we saw that this came down to checking that $\pm R$ satisfied one quadratic relation whose three coefficients were biquadratic forms in $\pm P$ and $\pm Q$. The same principle extends to Kummer surfaces, where the pseudo-group law is similarly defined by biquadratic forms; but since Kummer surfaces are defined in terms of four coordinates (as opposed to the two coordinates of the $x$-line), this time there are six simple quadratic relations to verify, with a total of ten coefficient forms.

\subsection{Biquadratic forms and pseudo-addition}

Let $\mathcal{K}$ be a Kummer surface. If $\pm P$ is a point on $\mathcal{K}$, then we write $\left(Z_{1}^{P}: Z_{2}^{P}: Z_{3}^{P}: Z_{4}^{P}\right)$ for its projective coordinates. The classical theory of abelian varieties tells us that there exist biquadratic forms $B_{i j}$ for $1 \leq i, j \leq 4$ such that for all $\pm P$ and $\pm Q$, if $\pm S= \pm(P+Q)$ and $\pm D= \pm(P-Q)$ then

$$
\left(Z_{i}^{S} Z_{j}^{D}+Z_{j}^{S} Z_{i}^{D}\right)_{i, j=1}^{4}=\lambda\left(B_{i j}\left(Z_{1}^{P}, Z_{2}^{P}, Z_{3}^{P}, Z_{4}^{P}, Z_{1}^{Q}, Z_{2}^{Q}, Z_{3}^{Q}, Z_{4}^{Q}\right)\right)_{i, j=1}^{4}
$$


where $\lambda \in \mathbb{k}^{\times}$is some common projective factor depending only on the affine representatives chosen for $\pm P, \pm Q, \pm(P+Q)$ and $\pm(P-Q)$. These biquadratic forms are the foundation of pseudo-addition and doubling laws on $\mathcal{K}$ : if the "difference" $\pm D$ is known, then we can use the $B_{i j}$ to compute $\pm S$.

Proposition 4. Let $\left\{B_{i j}: 1 \leq i, j \leq 4\right\}$ be a set of biquadratic forms on $\mathcal{K} \times \mathcal{K}$ satisfying Equation (11) for all $\pm P, \pm Q, \pm(P+Q)$, and $\pm(P-Q)$. Then

$$
\pm R=\left(Z_{1}^{R}: Z_{2}^{R}: Z_{3}^{R}: Z_{4}^{R}\right) \in\{ \pm(P+Q), \pm(P-Q)\}
$$

if and only if (writing $B_{i j}$ for $B_{i j}\left(Z_{1}^{P}, \ldots, Z_{4}^{Q}\right)$ ) we have

$$
B_{j j} \cdot\left(Z_{i}^{R}\right)^{2}-2 B_{i j} \cdot Z_{i}^{R} Z_{j}^{R}+B_{i i} \cdot\left(Z_{j}^{R}\right)^{2}=0 \quad \text { for all } 1 \leq i<j \leq 4 .
$$

Proof. Looking at Equation (11), we see that the system of six quadratics from Equation (12) cuts out a zero-dimensional degree- 2 subscheme of $\mathcal{K}$ : that is, the pair of points $\{ \pm(P+$ $Q), \pm(P-Q)\}$ (which may coincide). Hence, if $\left(Z_{1}^{R}: Z_{2}^{R}: Z_{3}^{R}: Z_{4}^{R}\right)= \pm R$ satisfies all of the equations, then it must be one of them.

\subsection{Deriving efficiently computable forms}

Proposition 4 is the exact analogue of Proposition 3 for Kummer surfaces. All that we need to turn it into a Check algorithm for qDSA is an explicit and efficiently computable representation of the $B_{i j}$. These forms depend on the projective model of the Kummer surface; so we write $B_{i j}^{\mathrm{Can}}, B_{i j}^{\mathrm{Sqr}}$, and $B_{i j}^{\mathrm{Int}}$ for the forms on the canonical, squared, and intermediate surfaces.

On the canonical surface, the forms $B_{i j}^{C a n}$ are classical (see e.g. [3, §2.2]). The on-diagonal forms $B_{i i}^{\text {Can }}$ are

$$
\begin{array}{ll}
B_{11}^{\text {Can }}=\frac{1}{4}\left(\frac{V_{1}}{\widehat{\mu}_{1}}+\frac{V_{2}}{\widehat{\mu}_{2}}+\frac{V_{3}}{\widehat{\mu}_{3}}+\frac{V_{4}}{\widehat{\mu}_{4}}\right), & B_{22}^{\text {Can }}=\frac{1}{4}\left(\frac{V_{1}}{\widehat{\mu}_{1}}+\frac{V_{2}}{\widehat{\mu}_{2}}-\frac{V_{3}}{\widehat{\mu}_{3}}-\frac{V_{4}}{\widehat{\mu}_{4}}\right), \\
B_{33}^{\text {Can }}=\frac{1}{4}\left(\frac{V_{1}}{\widehat{\mu}_{1}}-\frac{V_{2}}{\widehat{\mu}_{2}}+\frac{V_{3}}{\widehat{\mu}_{3}}-\frac{V_{4}}{\widehat{\mu}_{4}}\right), & B_{44}^{\text {Can }}=\frac{1}{4}\left(\frac{V_{1}}{\widehat{\mu}_{1}}-\frac{V_{2}}{\widehat{\mu}_{2}}-\frac{V_{3}}{\widehat{\mu}_{3}}+\frac{V_{4}}{\widehat{\mu}_{4}}\right),
\end{array}
$$

where

$$
\begin{aligned}
& V_{1}=\left(\left(T_{1}^{P}\right)^{2}+\left(T_{2}^{P}\right)^{2}+\left(T_{3}^{P}\right)^{2}+\left(T_{4}^{P}\right)^{2}\right)\left(\left(T_{1}^{Q}\right)^{2}+\left(T_{2}^{Q}\right)^{2}+\left(T_{3}^{Q}\right)^{2}+\left(T_{4}^{Q}\right)^{2}\right), \\
& V_{2}=\left(\left(T_{1}^{P}\right)^{2}+\left(T_{2}^{P}\right)^{2}-\left(T_{3}^{P}\right)^{2}-\left(T_{4}^{P}\right)^{2}\right)\left(\left(T_{1}^{Q}\right)^{2}+\left(T_{2}^{Q}\right)^{2}-\left(T_{3}^{Q}\right)^{2}-\left(T_{4}^{Q}\right)^{2}\right), \\
& V_{3}=\left(\left(T_{1}^{P}\right)^{2}-\left(T_{2}^{P}\right)^{2}+\left(T_{3}^{P}\right)^{2}-\left(T_{4}^{P}\right)^{2}\right)\left(\left(T_{1}^{Q}\right)^{2}-\left(T_{2}^{Q}\right)^{2}+\left(T_{3}^{Q}\right)^{2}-\left(T_{4}^{Q}\right)^{2}\right), \\
& V_{4}=\left(\left(T_{1}^{P}\right)^{2}-\left(T_{2}^{P}\right)^{2}-\left(T_{3}^{P}\right)^{2}+\left(T_{4}^{P}\right)^{2}\right)\left(\left(T_{1}^{Q}\right)^{2}-\left(T_{2}^{Q}\right)^{2}-\left(T_{3}^{Q}\right)^{2}+\left(T_{4}^{Q}\right)^{2}\right),
\end{aligned}
$$

while the off-diagonal forms $B_{i j}$ with $i \neq j$ are

$$
B_{i j}^{\text {Can }}=\frac{2}{\widehat{\mu}_{i} \widehat{\mu}_{j}-\widehat{\mu}_{k} \widehat{\mu}_{l}}\left(\begin{array}{c}
\alpha_{i} \alpha_{j}\left(T_{i}^{P} T_{j}^{P} T_{i}^{Q} T_{j}^{Q}+T_{k}^{P} T_{l}^{P} T_{k}^{Q} T_{l}^{Q}\right) \\
-\alpha_{k} \alpha_{l}\left(T_{i}^{P} T_{j}^{P} T_{k}^{Q} T_{l}^{Q}+T_{k}^{P} T_{l}^{P} T_{i}^{Q} T_{j}^{Q}\right)
\end{array}\right)
$$

where $\{i, j, k, l\}=\{1,2,3,4\}$.

All of these forms can be efficiently evaluated. The off-diagonal $B_{i j}^{\text {Can }}$ have a particularly compact shape, while the symmetry of the on-diagonal $B_{i i}^{\text {Can }}$ makes them particularly easy to 
compute simultaneously: indeed, that is exactly what we do in Gaudry's fast pseudo-addition algorithm for $\mathcal{K}^{\text {Can }}[21, \S 3.2]$.

Ideally, we would like to evaluate the $B_{i j}^{\mathrm{Sqr}}$ on $\mathcal{K}^{\mathrm{Sqr}}$, since that is where our inputs $\pm P$, $\pm Q$, and $\pm R$ live. We can compute the $B_{i j}^{\text {Sqr }}$ by dualizing the $B_{i j}^{\text {Can }}$, then pulling the $\widehat{B}_{i j}^{\text {Can }}$ on $\widehat{\mathcal{K}}^{\text {Can }}$ back to $\mathcal{K}^{\text {Sqr }}$ via $\widehat{\mathcal{C}} \circ \mathcal{H}$. But while the resulting on-diagonal $B_{i i}^{\text {Sqr }}$ maintain the symmetry and efficiency of the $B_{i i}^{\text {Can },}{ }^{6}$ the off-diagonal $B_{i j}^{\text {Sqr }}$ turn out to be much less pleasant, with less apparent exploitable symmetry. For our applications, this means that evaluating $B_{i j}^{\mathrm{Sqr}}$ for $i \neq j$ implies taking a significant hit in terms of stack and code size, not to mention time.

We could avoid this difficulty by mapping the inputs of Check from $\mathcal{K}^{\text {Sqr }}$ into $\widehat{\mathcal{K}}^{\text {Can }}$, and then evaluating the $\widehat{B}_{i j}^{\text {Can }}$. But this would involve using - and, therefore, storing - the four large unsquared $\widehat{\alpha}_{i}$, which is an important drawback.

Why do the nice $\widehat{B}_{i j}^{\text {Can }}$ become so ugly when pulled back to $\mathcal{K}^{\text {Sqr }}$ ? The map $\widehat{\mathcal{C}}: \mathcal{K}^{\text {Int }} \rightarrow \widehat{\mathcal{K}}^{\text {Can }}$ has no impact on the shape or number of monomials, so most of the ugliness is due to the Hadamard transform $\mathcal{H}: \mathcal{K}^{\text {Sqr }} \rightarrow \mathcal{K}^{\text {Int }}$. In particular, if we only pull back the $\widehat{B}_{i j}^{\text {Can }}$ as far as $\mathcal{K}^{\text {Int }}$, then the resulting $B_{i j}^{\text {Int }}$ retain the nice form of the $B_{i j}^{\text {Can }}$ but do not involve the $\widehat{\alpha}_{i}$. This fact prompts our solution: we map $\pm P, \pm Q$, and $\pm R$ through $\mathcal{H}$ onto $\mathcal{K}^{\text {Int }}$, and verify using the forms $B_{i j}^{\mathrm{Int}}$.

Theorem 1. Up to a common projective factor, the on-diagonal biquadratic forms on the intermediate surface $\mathcal{K}^{\text {Int }}$ are

$$
\begin{aligned}
& B_{11}^{\text {Int }}=\widehat{\mu}_{1}\left(\kappa_{1} F_{1}+\kappa_{2} F_{2}+\kappa_{3} F_{3}+\kappa_{4} F_{4}\right), \\
& B_{22}^{\text {Int }}=\widehat{\mu}_{2}\left(\kappa_{2} F_{1}+\kappa_{1} F_{2}+\kappa_{4} F_{3}+\kappa_{3} F_{4}\right), \\
& B_{33}^{\text {Int }}=\widehat{\mu}_{3}\left(\kappa_{3} F_{1}+\kappa_{4} F_{2}+\kappa_{1} F_{3}+\kappa_{2} F_{4}\right), \\
& B_{44}^{\text {Int }}=\widehat{\mu}_{4}\left(\kappa_{4} F_{1}+\kappa_{3} F_{2}+\kappa_{2} F_{3}+\kappa_{1} F_{4}\right),
\end{aligned}
$$

where

$$
\begin{array}{ll}
F_{1}=P_{1} Q_{1}+P_{2} Q_{2}+P_{3} Q_{3}+P_{4} Q_{4}, & F_{2}=P_{1} Q_{2}+P_{2} Q_{1}+P_{3} Q_{4}+P_{4} Q_{3}, \\
F_{3}=P_{1} Q_{3}+P_{3} Q_{1}+P_{2} Q_{4}+P_{4} Q_{2}, & F_{4}=P_{1} Q_{4}+P_{4} Q_{1}+P_{2} Q_{3}+P_{3} Q_{2},
\end{array}
$$

where $P_{i}=\widehat{\epsilon}_{i}\left(Y_{i}^{P}\right)^{2}$ and $Q_{i}=\widehat{\epsilon}_{i}\left(Y_{i}^{Q}\right)^{2}$ for $1 \leq i \leq 4$. Up to the same common projective factor, the off-diagonal forms are

$$
B_{i j}^{\mathrm{Int}}=C \cdot C_{i j} \cdot\left(\widehat{\mu}_{k} \widehat{\mu}_{l}\left(Y_{i j}^{P}-Y_{k l}^{P}\right)\left(Y_{i j}^{Q}-Y_{k l}^{Q}\right)+\left(\widehat{\mu}_{i} \widehat{\mu}_{j}-\widehat{\mu}_{k} \widehat{\mu}_{l}\right) Y_{k l}^{P} Y_{k l}^{Q}\right)
$$

for $\{i, j, k, l\}=\{1,2,3,4\}$ where $C_{i j}:=\widehat{\mu}_{i} \widehat{\mu}_{j}\left(\widehat{\mu}_{i} \widehat{\mu}_{k}-\widehat{\mu}_{j} \widehat{\mu}_{l}\right)\left(\widehat{\mu}_{i} \widehat{\mu}_{l}-\widehat{\mu}_{j} \widehat{\mu}_{k}\right), Y_{i j}^{P}:=Y_{i}^{P} Y_{j}^{P}$, $Y_{i j}^{Q}:=Y_{i}^{Q} Y_{j}^{Q}$, and

$$
C:=\frac{8\left(\mu_{1} \mu_{2} \mu_{3} \mu_{4}\right)\left(\widehat{\mu}_{1} \widehat{\mu}_{2} \widehat{\mu}_{3} \widehat{\mu}_{4}\right)}{\left(\widehat{\mu}_{1} \widehat{\mu}_{2}-\widehat{\mu}_{3} \widehat{\mu}_{4}\right)\left(\widehat{\mu}_{1} \widehat{\mu}_{3}-\widehat{\mu}_{2} \widehat{\mu}_{4}\right)\left(\widehat{\mu}_{1} \widehat{\mu}_{4}-\widehat{\mu}_{2} \widehat{\mu}_{3}\right)}
$$

\footnotetext{
${ }^{6}$ As they should, since they are the basis of the efficient pseudo-addition on $\mathcal{K}^{\mathrm{Sqr}}$ !
} 
Proof. By definition, $\widehat{T}_{i}^{S} \widehat{T}_{j}^{D}+\widehat{T}_{j}^{S} \widehat{T}_{i}^{D}=\widehat{B}_{i j}^{\text {Can }}\left(\widehat{T}_{1}^{P}, \ldots, \widehat{T}_{4}^{Q}\right)$. Pulling back via $\widehat{\mathcal{C}}$ using $\widehat{T}_{i}=Y_{i} / \widehat{\alpha}_{i}$ yields

$$
\begin{aligned}
B_{i j}^{\operatorname{Int}}\left(Y_{1}^{P}, \ldots, Y_{4}^{Q}\right)=Y_{i}^{S} Y_{j}^{D}+Y_{j}^{S} Y_{i}^{D} & =\widehat{\alpha}_{i} \widehat{\alpha}_{j}\left(\widehat{T}_{i}^{S} \widehat{T}_{j}^{D}+\widehat{T}_{j}^{S} \widehat{T}_{i}^{D}\right) \\
& =\widehat{\alpha}_{i} \widehat{\alpha}_{j} \cdot \widehat{B}_{i j}^{\text {Can }}\left(\widehat{T}_{1}^{P}, \ldots, \widehat{T}_{4}^{Q}\right) \\
& =\widehat{\alpha}_{i} \widehat{\alpha}_{j} \cdot \widehat{B}_{i j}^{\text {Can }}\left(Y_{1}^{P} / \widehat{\alpha}_{1}, \ldots, Y_{4}^{Q} / \widehat{\alpha}_{4}\right) .
\end{aligned}
$$

Dualizing the $B_{i j}^{\text {Can }}$ from Equations (13), (14), and (15), we find

$$
\begin{aligned}
& B_{11}^{\text {Int }}=\widehat{\mu}_{1} /\left(4 \mu_{1} \mu_{2} \mu_{3} \mu_{4}\left(\widehat{\mu}_{1} \widehat{\mu}_{2} \widehat{\mu}_{3} \widehat{\mu}_{4}\right)^{2}\right) \cdot\left(\kappa_{1} F_{1}+\kappa_{2} F_{2}+\kappa_{3} F_{3}+\kappa_{4} F_{4}\right), \\
& B_{22}^{\text {Int }}=\widehat{\mu}_{2} /\left(4 \mu_{1} \mu_{2} \mu_{3} \mu_{4}\left(\widehat{\mu}_{1} \widehat{\mu}_{2} \widehat{\mu}_{3} \widehat{\mu}_{4}\right)^{2}\right) \cdot\left(\kappa_{2} F_{1}+\kappa_{1} F_{2}+\kappa_{4} F_{3}+\kappa_{3} F_{4}\right), \\
& B_{33}^{\text {Int }}=\widehat{\mu}_{3} /\left(4 \mu_{1} \mu_{2} \mu_{3} \mu_{4}\left(\widehat{\mu}_{1} \widehat{\mu}_{2} \widehat{\mu}_{3} \widehat{\mu}_{4}\right)^{2}\right) \cdot\left(\kappa_{3} F_{1}+\kappa_{4} F_{2}+\kappa_{1} F_{3}+\kappa_{2} F_{4}\right), \\
& B_{44}^{\text {Int }}=\widehat{\mu}_{4} /\left(4 \mu_{1} \mu_{2} \mu_{3} \mu_{4}\left(\widehat{\mu}_{1} \widehat{\mu}_{2} \widehat{\mu}_{3} \widehat{\mu}_{4}\right)^{2}\right) \cdot\left(\kappa_{4} F_{1}+\kappa_{3} F_{2}+\kappa_{2} F_{3}+\kappa_{1} F_{4}\right),
\end{aligned}
$$

while the off-diagonal forms $B_{i j}$ with $i \neq j$ are

$$
B_{i j}^{\text {Int }}=\frac{2}{\widehat{\mu}_{k} \widehat{\mu}_{l}\left(\widehat{\mu}_{i} \widehat{\mu}_{j}-\widehat{\mu}_{k} \widehat{\mu}_{l}\right)}\left(\begin{array}{c}
\widehat{\mu}_{k} \widehat{\mu}_{l}\left(Y_{i j}^{P}-Y_{k l}^{P}\right)\left(Y_{i j}^{Q}-Y_{k l}^{Q}\right) \\
+\left(\widehat{\mu}_{i} \widehat{\mu}_{j}-\widehat{\mu}_{k} \widehat{\mu}_{l}\right) Y_{k l}^{P} Y_{k l}^{Q}
\end{array}\right)
$$

for $\{i, j, k, l\}=\{1,2,3,4\}$. Multiplying all of these forms by a common projective factor of $4\left(\mu_{1} \mu_{2} \mu_{3} \mu_{4}\right)\left(\widehat{\mu}_{1} \widehat{\mu}_{2} \widehat{\mu}_{3} \widehat{\mu}_{4}\right)^{2}$ eliminates the denominators in the coefficients, and yields the forms of the theorem.

\subsection{Signature verification}

We are now finally ready to implement the Check algorithm for $\mathcal{K}^{\text {Sqr }}$. Algorithm 3 does this by applying $\mathcal{H}$ to its inputs, then using the biquadratic forms of Theorem 1 . Its correctness is implied by Proposition 4 .

\subsection{Using cryptographic parameters}

Gaudry and Schost take $p=2^{127}-1$ and $\left(\mu_{1}: \mu_{2}: \mu_{3}: \mu_{4}\right)=(-11: 22: 19: 3)$ in [22]. We also need the constants $\left(\widehat{\mu}_{1}: \widehat{\mu}_{2}: \widehat{\mu}_{3}: \widehat{\mu}_{4}\right)=(-33: 11: 17: 49),\left(\kappa_{1}: \kappa_{2}: \kappa_{3}: \kappa_{4}\right)=(-4697:$ $5951: 5753:-1991)$, and $\left(\widehat{\epsilon}_{1}: \widehat{\epsilon}_{2}: \widehat{\epsilon}_{3}: \widehat{\epsilon}_{4}\right)=(-833: 2499: 1617: 561){ }^{7}$ In practice, where these constants are "negative", we reverse their sign and amend the formulæ above accordingly. All of these constants are small, and fit into one or two bytes each (and the $\widehat{\epsilon}_{i}$ are already stored for use in Ladder). We store one large constant

$$
C=\text { 0x40F50EEFA320A2DD46F7E3D8CDDDA843, }
$$

and recompute the $C_{i j}$ on the fly.

\footnotetext{
${ }^{7}$ Following the definitions of $\S 4.1$, the $\widehat{\mu}_{i}$ are scaled by -2 , the $\widehat{\epsilon}_{i}$ by $1 / 11$, and $C$ by $2 / 11^{2}$. These changes influence the $B_{i j}^{\text {Int }}$, but only up to the same projective factor.
} 


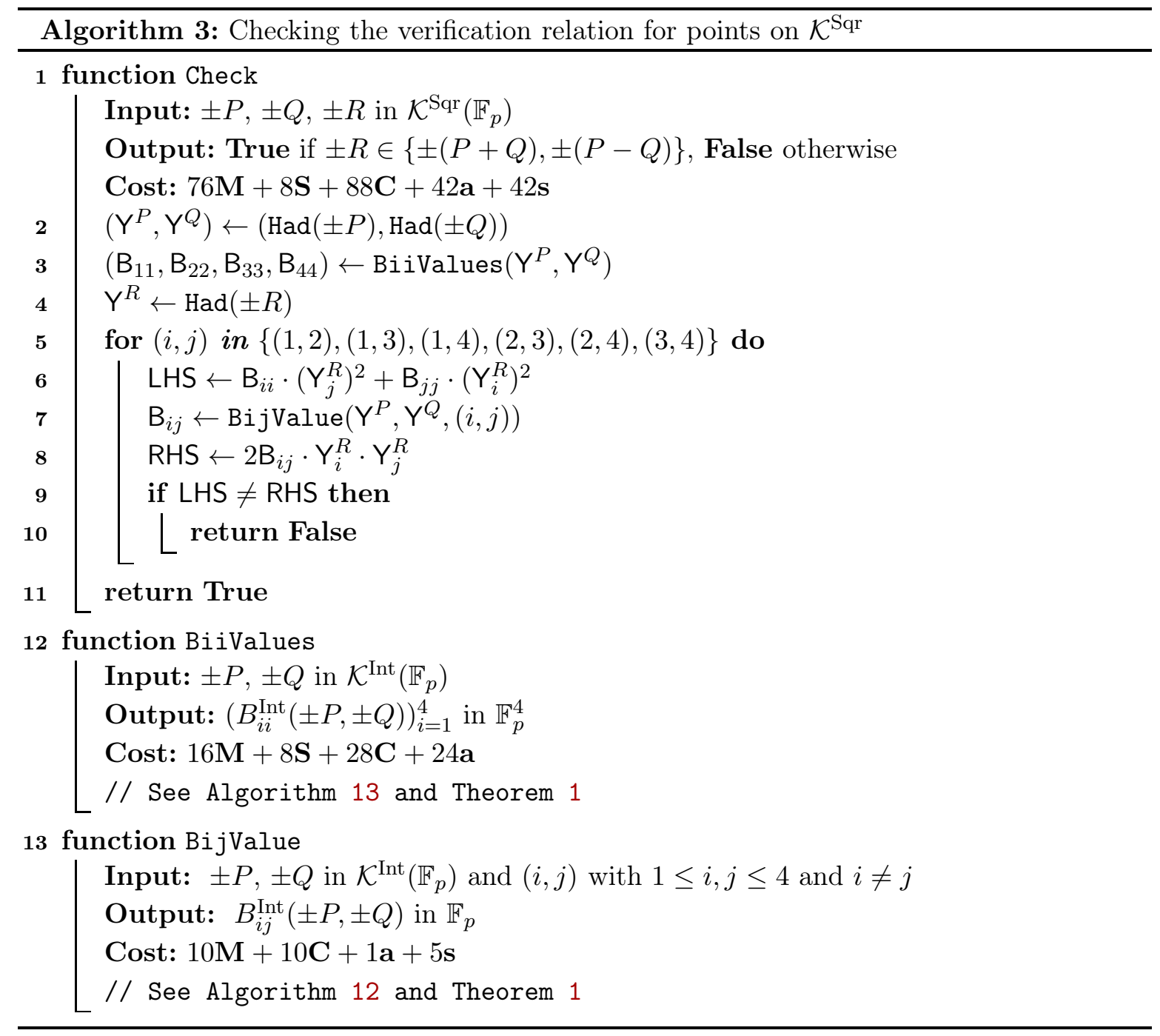

\section{Kummer point compression}

Our public keys are points on $\mathcal{K}^{\mathrm{Sqr}}$, and each signature includes one point on $\mathcal{K}^{\mathrm{Sqr}}$. Minimizing the space required by Kummer points is therefore essential.

A projective Kummer point is composed of four field elements; normalizing by dividing through by a nonzero coordinate reduces us to three field elements (this can also be achieved using Bernstein's "wrapping" technique [6], as in [7] and [39]). But we are talking about Kummer surfaces - two-dimensional objects - so we might hope to compress to two field elements, plus a few bits to enable us to correctly recover the whole Kummer point. This is analogous to elliptic curve point compression, where we compress projective points ( $X: Y: Z)$ by normalizing to $(x, y)=(X / Z, Y / Z)$, then storing $(x, \sigma)$, where $\sigma$ is a bit indicating the "sign" of $y$. Decompressing the datum $(x, \sigma)$ to $(X: Y: Z)=(x: y: 1)$ then requires solving a simple quadratic to recover the correct $y$-coordinate.

For some reason, no such Kummer point compression method has explicitly appeared in the literature. Bernstein remarked in 2006 that if we compress a Kummer point to two co- 
ordinates, then decompression appears to require solving a complicated quartic equation [6]. This would be much more expensive than computing the single square root required for elliptic decompression; this has perhaps discouraged implementers from attempting to compress Kummer points.

But while it may not always be obvious from their defining equations, the classical theory tells us that every Kummer is in fact a double cover of $\mathbb{P}^{2}$, just as elliptic curves are double covers of $\mathbb{P}^{1}$. We use this principle below to show that we can always compress any Kummer point to two field elements plus two auxiliary bits, and then decompress by solving a quadratic. In our applications, this gives us a convenient packaging of Kummer points in exactly 256 bits.

\subsection{The general principle}

First, we sketch a general method for Kummer point compression that works for any Kummer presented as a singular quartic surface in $\mathbb{P}^{3}$.

Recall that if $N$ is any point in $\mathbb{P}^{3}$, then projection away from $N$ defines a map $\pi_{N}: \mathbb{P}^{3} \rightarrow$ $\mathbb{P}^{2}$ sending points in $\mathbb{P}^{3}$ on the same line through $N$ to the same point in $\mathbb{P}^{2}$. (The map $\pi_{N}$ is only a rational map, and not a morphism; the image of $N$ itself is not well-defined.) Now, let $N$ be a node of a Kummer surface $\mathcal{K}$ : that is, $N$ is one of the 16 singular points of $\mathcal{K}$. The restriction of $\pi_{N}$ to $\mathcal{K}$ forms a double cover of $\mathbb{P}^{2}$. By definition, $\pi_{N}$ maps the points on $\mathcal{K}$ that lie on the same line through $N$ to the same point of $\mathbb{P}^{2}$. Now $\mathcal{K}$ has degree 4 , so each line in $\mathbb{P}^{3}$ intersects $\mathcal{K}$ in four points; but since $N$ is a double point of $\mathcal{K}$, every line through $N$ intersects $\mathcal{K}$ at $N$ twice, and then in two other points. These two remaining points may be "compressed" to their common image in $\mathbb{P}^{2}$ under $\pi_{N}$, plus a single bit to distinguish the appropriate preimage.

To make this more concrete, let $L_{1}, L_{2}$, and $L_{3}$ be linearly independent linear forms on $\mathbb{P}^{3}$ vanishing on $N$; then $N$ is the intersection of the three planes in $\mathbb{P}^{3}$ cut out by the $L_{i}$. We can now realise the projection $\pi_{N}: \mathcal{K} \rightarrow \mathbb{P}^{2}$ as

$$
\pi_{N}:\left(P_{1}: \cdots: P_{4}\right) \longmapsto\left(L_{1}\left(P_{1}, \ldots, P_{4}\right): L_{2}\left(P_{1}, \ldots, P_{4}\right): L_{3}\left(P_{1}, \ldots, P_{4}\right)\right) .
$$

Replacing $\left(L_{1}, L_{2}, L_{3}\right)$ with another basis of $\left\langle L_{1}, L_{2}, L_{3}\right\rangle$ yields another projection, which corresponds to composing $\pi_{N}$ with a linear automorphism of $\mathbb{P}^{2}$.

If $L_{1}, L_{2}$, and $L_{3}$ are chosen as above to vanish on $N$, and $L_{4}$ is any linear form not in $\left\langle L_{1}, L_{2}, L_{3}\right\rangle$, then the fact that $\pi_{N}$ is a double cover of the $\left(L_{1}, L_{2}, L_{3}\right)$-plane implies that the defining equation of $\mathcal{K}$ can be rewritten in the form

$$
\mathcal{K}: K_{2}\left(L_{1}, L_{2}, L_{3}\right) L_{4}^{2}-2 K_{3}\left(L_{1}, L_{2}, L_{3}\right) L_{4}+K_{4}\left(L_{1}, L_{2}, L_{3}\right)=0
$$

where each $K_{i}$ is a homogeneous polynomial of degree $i$ in $L_{1}, L_{2}$, and $L_{3}$. This form, quadratic in $L_{4}$, allows us to replace the $L_{4}$-coordinate with a single bit indicating the "sign" in the corresponding root of this quadratic; the remaining three coordinates can be normalized to an affine plane point. The net result is a compression to two field elements, plus one bit indicating the normalization, plus another bit to indicate the correct value of $L_{4}$.

Remark 1. Stahlke gives a compression algorithm in [42] for points on genus-2 Jacobians in the usual Mumford representation. The first step can be seen as a projection to the most general model of the Kummer (as in [12, Chapter 3]), and then the second is an implicit implementation of the principle above. 


\subsection{From squared Kummers to tetragonal Kummers}

We want to define an efficient point compression scheme for $\mathcal{K}^{\mathrm{Sqr}}$. The general principle above makes this possible, but it leaves open the choice of node $N$ and the choice of forms $L_{i}$. These choices determine the complexity of the resulting $K_{i}$, and hence the cost of evaluating them; this in turn has a non-negligible impact on the time and space required to compress and decompress points, as well as the number of new auxiliary constants that must be stored.

In this section we define a choice of $L_{i}$ reflecting the special symmetry of $\mathcal{K}^{\mathrm{Sqr}}$. A similar procedure for $\mathcal{K}^{\text {Can }}$ appears in more classical language ${ }^{8}$ in $[26, \S 54]$. The trick is to distinguish not one node of $\mathcal{K}^{\mathrm{Sqr}}$, but rather the four nodes forming the kernel of the $(2,2)$-isogeny $\widehat{\mathcal{S}} \circ \widehat{\mathcal{C}} \circ \mathcal{H}: \mathcal{K}^{\mathrm{Sqr}} \rightarrow \widehat{\mathcal{K}}^{\mathrm{Sqr}}$, namely

$$
\begin{aligned}
\pm 0= & N_{0}=\left(\mu_{1}: \mu_{2}: \mu_{3}: \mu_{4}\right), & & N_{1}=\left(\mu_{2}: \mu_{1}: \mu_{4}: \mu_{3}\right), \\
& N_{2}=\left(\mu_{3}: \mu_{4}: \mu_{1}: \mu_{2}\right), & & N_{3}=\left(\mu_{4}: \mu_{3}: \mu_{2}: \mu_{1}\right) .
\end{aligned}
$$

We are going to define a coordinate system where these four nodes become the vertices of a coordinate tetrahedron; then, projection onto any three of the four coordinates will represent a projection away from one of these four nodes. The result will be an isomorphic Kummer $\mathcal{K}^{\text {Tet }}$ whose defining equation is quadratic in all four of its variables. This might seem like overkill for point compression - quadratic in just one variable would suffice-but it has the agreeable effect of dramatically reducing the overall complexity of the defining equation, saving time and memory in our compression and decompression algorithms.

The key is the matrix identity

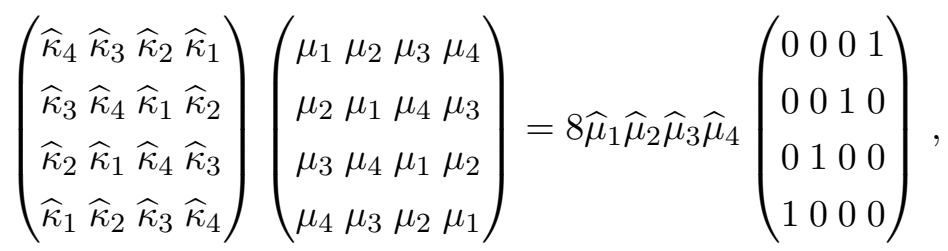

which tells us that the projective isomorphism $\mathcal{T}: \mathbb{P}^{3} \rightarrow \mathbb{P}^{3}$ defined by

$$
\mathcal{T}:\left(\begin{array}{c}
X_{1} \\
: X_{2} \\
: X_{3} \\
: X_{4}
\end{array}\right) \mapsto\left(\begin{array}{c}
L_{1} \\
: L_{2} \\
: L_{3} \\
: L_{4}
\end{array}\right)=\left(\begin{array}{c}
\widehat{\kappa}_{4} X_{1}+\widehat{\kappa}_{3} X_{2}+\widehat{\kappa}_{2} X_{3}+\widehat{\kappa}_{1} X_{4} \\
: \widehat{\kappa}_{3} X_{1}+\widehat{\kappa}_{4} X_{2}+\widehat{\kappa}_{1} X_{3}+\widehat{\kappa}_{2} X_{4} \\
: \widehat{\kappa}_{2} X_{1}+\widehat{\kappa}_{1} X_{2}+\widehat{\kappa}_{4} X_{3}+\widehat{\kappa}_{3} X_{4} \\
: \widehat{\kappa}_{1} X_{1}+\widehat{\kappa}_{2} X_{2}+\widehat{\kappa}_{3} X_{3}+\widehat{\kappa}_{4} X_{4}
\end{array}\right)
$$

maps the four "kernel" nodes to the corners of a coordinate tetrahedron:

$$
\begin{array}{ll}
\mathcal{T}\left(N_{0}\right)=(0: 0: 0: 1), & \mathcal{T}\left(N_{2}\right)=(0: 1: 0: 0), \\
\mathcal{T}\left(N_{1}\right)=(0: 0: 1: 0), & \mathcal{T}\left(N_{3}\right)=(1: 0: 0: 0) .
\end{array}
$$

\footnotetext{
${ }^{8}$ The analogous model of $\mathcal{K}^{\text {Can }}$ in $[26, \S 54]$ is called "the equation referred to a Rosenhain tetrad", whose defining equation "...may be deduced from the fact that Kummer's surface is the focal surface of the congruence of rays common to a tetrahedral complex and a linear complex." Modern cryptographers will understand why we have chosen to give a little more algebraic detail here.
} 
The image of $\mathcal{K}^{\mathrm{Sqr}}$ under $\mathcal{T}$ is the tetragonal surface

$$
\mathcal{K}^{\mathrm{Tet}}: 4 t L_{1} L_{2} L_{3} L_{4}=\begin{aligned}
r_{1}^{2}\left(L_{1} L_{2}\right. & \left.+L_{3} L_{4}\right)^{2}+r_{2}^{2}\left(L_{1} L_{3}+L_{2} L_{4}\right)^{2}+r_{3}^{2}\left(L_{1} L_{4}+L_{2} L_{3}\right)^{2} \\
& -2 r_{1} s_{1}\left(\left(L_{1}^{2}+L_{2}^{2}\right) L_{3} L_{4}+L_{1} L_{2}\left(L_{3}^{2}+L_{4}^{2}\right)\right) \\
& -2 r_{2} s_{2}\left(\left(L_{1}^{2}+L_{3}^{2}\right) L_{2} L_{4}+L_{1} L_{3}\left(L_{2}^{2}+L_{4}^{2}\right)\right) \\
& -2 r_{3} s_{3}\left(\left(L_{1}^{2}+L_{4}^{2}\right) L_{2} L_{3}+L_{1} L_{4}\left(L_{2}^{2}+L_{3}^{2}\right)\right)
\end{aligned}
$$

where $t=16 \mu_{1} \mu_{2} \mu_{3} \mu_{4} \widehat{\mu}_{1} \widehat{\mu}_{2} \widehat{\mu}_{3} \widehat{\mu}_{4}$ and

$$
\begin{array}{ll}
r_{1}=\left(\mu_{1} \mu_{3}-\mu_{2} \mu_{4}\right)\left(\mu_{1} \mu_{4}-\mu_{2} \mu_{3}\right), & s_{1}=\left(\mu_{1} \mu_{2}-\mu_{3} \mu_{4}\right)\left(\mu_{1} \mu_{2}+\mu_{3} \mu_{4}\right), \\
r_{2}=\left(\mu_{1} \mu_{2}-\mu_{3} \mu_{4}\right)\left(\mu_{1} \mu_{4}-\mu_{2} \mu_{3}\right), & s_{2}=\left(\mu_{1} \mu_{3}-\mu_{2} \mu_{4}\right)\left(\mu_{1} \mu_{3}+\mu_{2} \mu_{4}\right), \\
r_{3}=\left(\mu_{1} \mu_{2}-\mu_{3} \mu_{4}\right)\left(\mu_{1} \mu_{3}-\mu_{2} \mu_{4}\right), & s_{3}=\left(\mu_{1} \mu_{4}-\mu_{2} \mu_{3}\right)\left(\mu_{1} \mu_{4}+\mu_{2} \mu_{3}\right) .
\end{array}
$$

As promised, the defining equation of $\mathcal{K}^{\text {Tet }}$ is quadratic in all four of its variables.

For compression we project away from $\mathcal{T}( \pm 0)=(0: 0: 0: 1)$ onto the $\left(L_{1}: L_{2}: L_{3}\right)$-plane. Rewriting the defining equation as a quadratic in $L_{4}$ gives

$$
\mathcal{K}^{\mathrm{Tet}}: K_{4}\left(L_{1}, L_{2}, L_{3}\right)-2 K_{3}\left(L_{1}, L_{2}, L_{3}\right) L_{4}+K_{2}\left(L_{1}, L_{2}, L_{3}\right) L_{4}^{2}=0
$$

where

$$
\begin{aligned}
K_{2}:= & r_{3}^{2} L_{1}^{2}+r_{2}^{2} L_{2}^{2}+r_{1}^{2} L_{3}^{2}-2\left(r_{3} s_{3} L_{2} L_{3}+r_{2} s_{2} L_{1} L_{3}+r_{1} s_{1} L_{1} L_{2}\right), \\
K_{3}:= & r_{1} s_{1}\left(L_{1}^{2}+L_{2}^{2}\right) L_{3}+r_{2} s_{2}\left(L_{1}^{2}+L_{3}^{2}\right) L_{2}+r_{3} s_{3}\left(L_{2}^{2}+L_{3}^{2}\right) L_{1} \\
& \quad+\left(2 t-\left(r_{1}^{2}+r_{2}^{2}+r_{3}^{2}\right)\right) L_{1} L_{2} L_{3}, \\
K_{4}:= & r_{3}^{2} L_{2}^{2} L_{3}^{2}+r_{2}^{2} L_{1}^{2} L_{3}^{2}+r_{1}^{2} L_{1}^{2} L_{2}^{2}-2\left(r_{3} s_{3} L_{1}+r_{2} s_{2} L_{2}+r_{1} s_{1} L_{3}\right) L_{1} L_{2} L_{3} .
\end{aligned}
$$

Lemma 1. If $\left(l_{1}: l_{2}: l_{3}: l_{4}\right)$ is a point on $\mathcal{K}^{\text {Tet }}$, then

$$
K_{2}\left(l_{1}, l_{2}, l_{3}\right)=K_{3}\left(l_{1}, l_{2}, l_{3}\right)=K_{4}\left(l_{1}, l_{2}, l_{3}\right)=0 \Longleftrightarrow l_{1}=l_{2}=l_{3}=0 .
$$

Proof. Write $k_{i}$ for $K_{i}\left(l_{1}, l_{2}, l_{3}\right)$. If $\left(l_{1}, l_{2}, l_{3}\right)=0$ then $\left(k_{2}, k_{3}, k_{4}\right)=0$, because each $K_{i}$ is nonconstant and homogeneous. Conversely, if $\left(k_{2}, k_{3}, k_{4}\right)=0$ and $\left(l_{1}, l_{2}, l_{3}\right) \neq 0$ then we could embed a line in $\mathcal{K}^{\text {Tet }}$ via $\lambda \mapsto\left(l_{1}: l_{2}: l_{3}: \lambda\right)$; but this is a contradiction, because $\mathcal{K}^{\text {Tet }}$ contains no lines.

\subsection{Compression and decompression for $\mathcal{K}^{\mathrm{Sqr}}$}

In practice, we compress points on $\mathcal{K}^{\mathrm{Sqr}}$ to tuples $\left(l_{1}, l_{2}, \tau, \sigma\right)$, where $l_{1}$ and $l_{2}$ are field elements and $\tau$ and $\sigma$ are bits. The recipe is

(1) Map $\left(X_{1}: X_{2}: X_{3}: X_{4}\right)$ through $\mathcal{T}$ to a point $\left(L_{1}: L_{2}: L_{3}: L_{4}\right)$ on $\mathcal{K}^{\mathrm{Tet}}$.

(2) Compute the unique $\left(l_{1}, l_{2}, l_{3}, l_{4}\right)$ in one of the forms $(*, *, 1, *),(*, 1,0, *),(1,0,0, *)$, or $(0,0,0,1)$ such that $\left(l_{1}: l_{2}: l_{3}: l_{4}\right)=\left(L_{1}: L_{2}: L_{3}: L_{4}\right)$.

(3) Compute $k_{2}=K_{2}\left(l_{1}, l_{2}, l_{3}\right), k_{3}=K_{3}\left(l_{1}, l_{2}, l_{3}\right)$, and $k_{4}=K_{4}\left(l_{1}, l_{2}, l_{3}\right)$.

(4) Define the bit $\sigma=\operatorname{Sign}\left(k_{2} l_{4}-k_{3}\right)$; then $\left(l_{1}, l_{2}, l_{3}, \sigma\right)$ determines $l_{4}$. Indeed, $q\left(l_{4}\right)=0$, where $q(X)=k_{2} X^{2}-2 k_{3} X+k_{4}$; and Lemma 1 tells us that $q(X)$ is either quadratic, linear, or identically zero. 
- If $q$ is a nonsingular quadratic, then $l_{4}$ is determined by $\left(l_{1}, l_{2}, l_{3}\right)$ and $\sigma$, because $\sigma=\operatorname{Sign}(R)$ where $R$ is the correct square root in the quadratic formula $l_{4}=\left(k_{3} \pm\right.$ $\left.\sqrt{k_{3}^{2}-k_{2} k_{4}}\right) / k_{2}$.

- If $q$ is singular or linear, then $\left(l_{1}, l_{2}, l_{3}\right)$ determines $l_{4}$, and $\sigma$ is redundant.

- If $q=0$ then $\left(l_{1}, l_{2}, l_{3}\right)=(0,0,0)$, so $l_{4}=1$; again, $\sigma$ is redundant.

Setting $\sigma=\operatorname{Sign}\left(k_{2} l_{4}-k_{3}\right)$ in every case, regardless of whether or not we need it to determine $l_{4}$, avoids ambiguity and simplifies code.

(5) The normalization in Step 2 forces $l_{3} \in\{0,1\}$; so encode $l_{3}$ as a single bit $\tau$.

The datum $\left(l_{1}, l_{2}, \tau, \sigma\right)$ completely determines $\left(l_{1}, l_{2}, l_{3}, l_{4}\right)$, and thus determines $\left(X_{1}: X_{2}\right.$ : $\left.X_{3}: X_{4}\right)=\mathcal{T}^{-1}\left(\left(l_{1}: l_{2}: l_{2}: l_{4}\right)\right)$. Conversely, the normalization in Step 2 ensures that $\left(l_{1}, l_{2}, \tau, \sigma\right)$ is uniquely determined by $\left(X_{1}: X_{2}: X_{3}: X_{4}\right)$, and is independent of the representative values of the $X_{i}$.

Algorithm 4 carries out the compression process above; the most expensive step is the computation of an inverse in $\mathbb{F}_{p}$. Algorithm 5 is the corresponding decompression algorithm; its cost is dominated by computing a square root in $\mathbb{F}_{p}$.

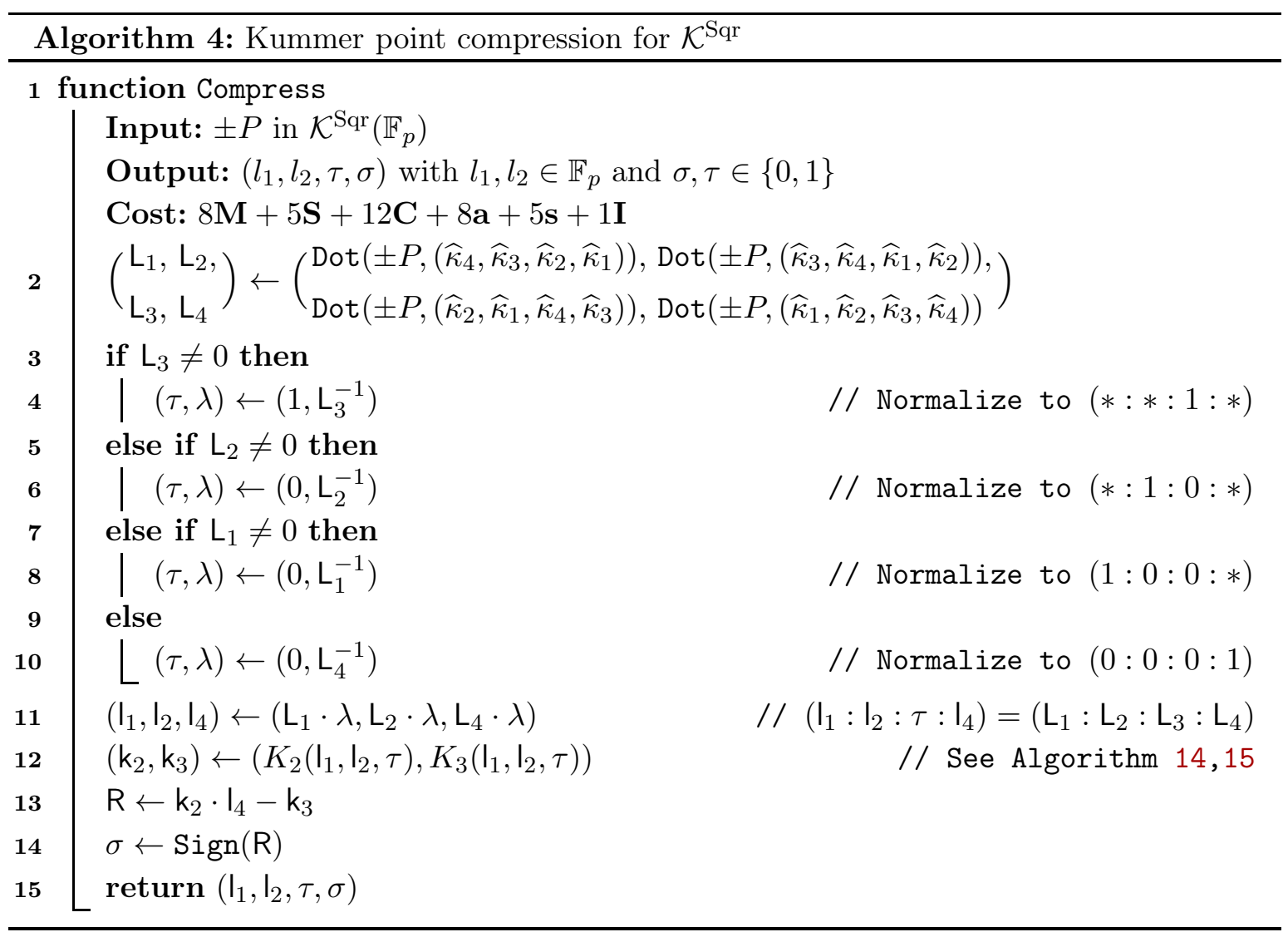

Proposition 5. Algorithms 4 and 5 (Compress and Decompress) satisfy the following properties: given $\left(l_{1}, l_{2}, \tau, \sigma\right)$ in $\mathbb{F}_{p}^{2} \times\{0,1\}^{2}$, Decompress always returns either a valid point in $\mathcal{K}^{\mathrm{Sqr}}\left(\mathbb{F}_{p}\right)$ or $\perp$; and for every $\pm P$ in $\mathcal{K}^{\mathrm{Sqr}}\left(\mathbb{F}_{p}\right)$, we have

$$
\text { Decompress(Compress }( \pm P))= \pm P \text {. }
$$




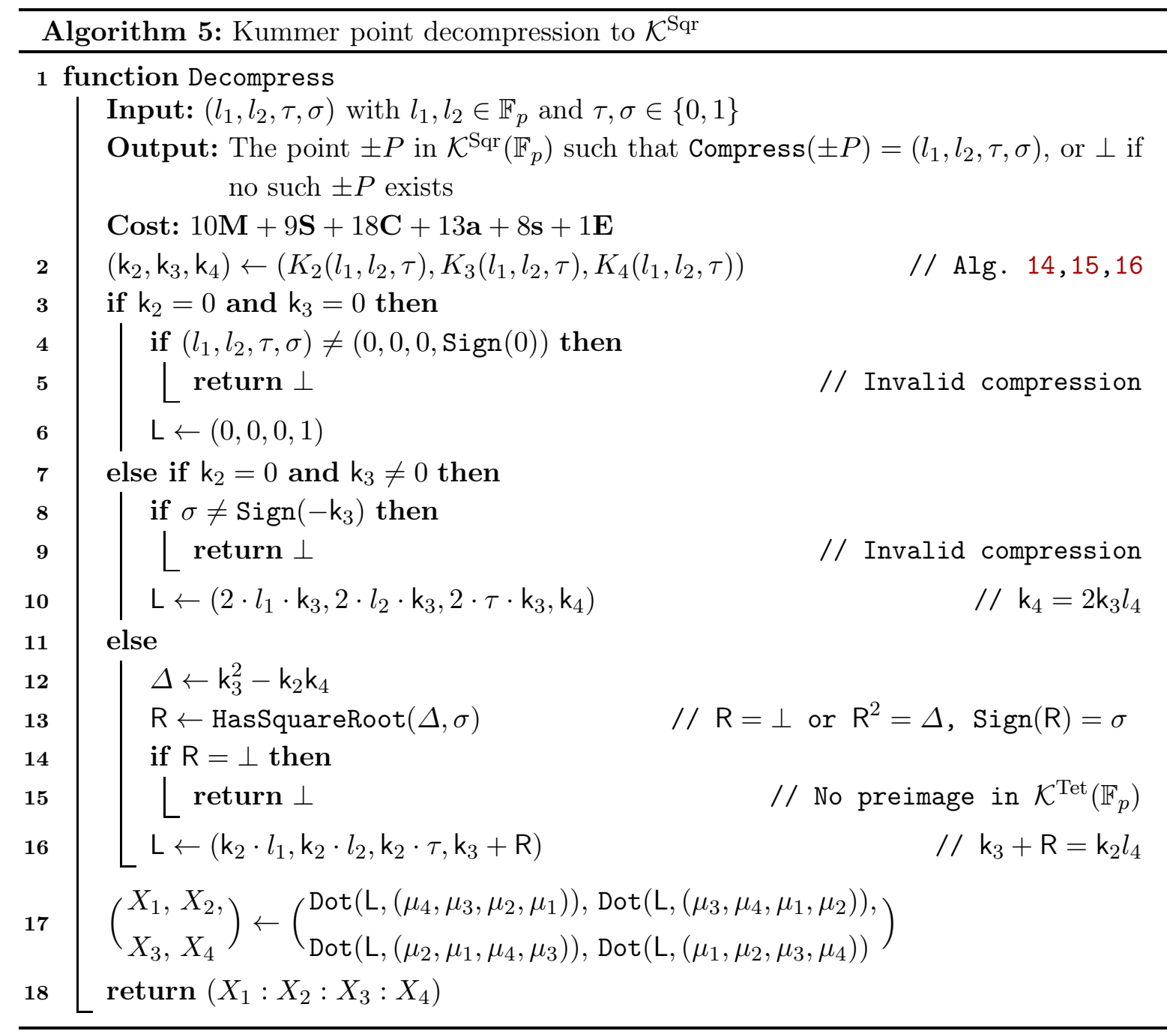

Proof. In Algorithm 5 we are given $\left(l_{1}, l_{2}, \tau, \sigma\right)$. We can immediately set $l_{3}=\tau$, viewed as an element of $\mathbb{F}_{p}$. We want to compute an $l_{4}$ in $\mathbb{F}_{p}$, if it exists, such that $k_{2} l_{4}^{2}-2 k_{3} l_{4}+k_{4}=0$ and $\operatorname{Sign}\left(k_{2} l_{4}-l_{3}\right)=\sigma$ where $k_{i}=K_{i}\left(l_{1}, l_{2}, l_{3}\right)$. If such an $l_{4}$ exists, then we will have a preimage $\left(l_{1}: l_{2}: l_{3}: l_{4}\right)$ in $\mathcal{K}^{\mathrm{Tet}}\left(\mathbb{F}_{p}\right)$, and we can return the decompressed $\mathcal{T}^{-1}\left(\left(l_{1}: l_{2}: l_{3}: l_{4}\right)\right)$ in $\mathcal{K}^{\mathrm{Sqr}}$.

If $\left(k_{2}, k_{3}\right)=(0,0)$ then $k_{4}=2 k_{3} l_{4}-k_{2} l_{4}^{2}=0$, so $l_{1}=l_{2}=\tau=0$ by Lemma 1 . The only legitimate datum in this form is is $\left(l_{1}: l_{2}: \tau: \sigma\right)=(0: 0: 0: \operatorname{Sign}(0))$. If this was the input, then the preimage is $(0: 0: 0: 1)$; otherwise we return $\perp$.

If $k_{2}=0$ but $k_{3} \neq 0$, then $k_{4}=2 k_{3} l_{4}$, so $\left(l_{1}: l_{2}: \tau: l_{4}\right)=\left(2 k_{3} l_{1}: 2 k_{3} l_{2}: 2 k_{3} \tau: k_{4}\right)$. The datum is a valid compression unless $\sigma \neq \operatorname{Sign}\left(-k_{3}\right)$, in which case we return $\perp$; otherwise, the preimage is $\left(2 k_{3} l_{1}: 2 k_{3} l_{2}: 2 k_{3} \tau: k_{4}\right)$.

If $k_{2} \neq 0$, then the quadratic formula tells us that any preimage satisfies $k_{2} l_{4}=k_{3} \pm$ $\sqrt{k_{3}^{2}-k_{2} k_{4}}$, with the sign determined by $\operatorname{Sign}\left(k_{2} l_{4}-k_{3}\right)$. If $k_{3}^{2}-k_{2} k_{4}$ is not a square in $\mathbb{F}_{p}$ then there is no such $l_{4}$ in $\mathbb{F}_{p}$; the input is illegitimate, so we return $\perp$. Otherwise, we have a preimage $\left(k_{2} l_{1}: k_{2} l_{2}: k_{2} l_{3}: l_{3} \pm \sqrt{k_{3}^{2}-k_{2} k_{4}}\right)$. 
Line 17 maps the preimage $\left(l_{1}: l_{2}: l_{3}: l_{4}\right)$ in $\mathcal{K}^{\text {Tet }}\left(\mathbb{F}_{p}\right)$ back to $\mathcal{K}^{\mathrm{Sqr}}\left(\mathbb{F}_{p}\right)$ via $\mathcal{T}^{-1}$, yielding the decompressed point $\left(X_{1}: X_{2}: X_{3}: X_{4}\right)$.

\subsection{Using cryptographic parameters}

Our compression scheme works out particularly nicely for the Gaudry-Schost Kummer over $\mathbb{F}_{2^{127}-1}$. First, since every field element fits into 127 bits, every compressed point fits into exactly 256 bits. Second, the auxiliary constants are small: we have $\left(\widehat{\kappa}_{1}: \widehat{\kappa}_{2}: \widehat{\kappa}_{3}: \widehat{\kappa}_{4}\right)=$ $(-961: 128: 569: 1097)$, each of which fits into well under 16 bits. Computing the polynomials $K_{2}, K_{3}, K_{4}$ and dividing them all through by $11^{2}$ (which does not change the roots of the quadratic) gives

$$
\begin{aligned}
& K_{2}\left(l_{1}, l_{2}, \tau\right)=\left(q_{5} l_{1}\right)^{2}+\left(q_{3} l_{2}\right)^{2}+\left(q_{4} \tau\right)^{2}-2 q_{3}\left(q_{2} l_{1} l_{2}+\tau\left(q_{0} l_{1}-q_{1} l_{2}\right)\right), \\
& K_{3}\left(l_{1}, l_{2}, \tau\right)=q_{3}\left(q_{0}\left(l_{1}^{2}+\tau\right) l_{2}-q_{1} l_{1}\left(l_{2}^{2}+\tau\right)+q_{2}\left(l_{1}^{2}+l_{2}^{2}\right) \tau\right)-q_{6} q_{7} l_{1} l_{2} \tau, \\
& K_{4}\left(l_{1}, l_{2}, \tau\right)=\left(\left(q_{3} l_{1}\right)^{2}+\left(q_{5} l_{2}\right)^{2}-2 q_{3} l_{1} l_{2}\left(q_{0} l_{2}-q_{1} l_{1}+q_{2}\right)\right) \tau+\left(q_{4} l_{1} l_{2}\right)^{2},
\end{aligned}
$$

where $\left(q_{0}, \ldots, q_{7}\right)=(3575,9625,4625,12259,11275,7475,6009,43991)$; each of the $q_{i}$ fits into 16 bits. In total, the twelve new constants we need for Compress and Decompress together fit into less than two field elements' worth of space.

\section{Implementation}

In this section we present the results of the implementation of the scheme on the AVR ATmega and ARM Cortex M0 platforms. We have a total of four implementations: on both platforms we implemented both the Curve25519-based scheme and the scheme based on a fast Kummer surface in genus 2. The benchmarks for the AVR software are obtained from the Arduino MEGA development board containing an ATmega2560 MCU, compiled with GCC v4.8.1. For the Cortex M0, they are measured on the STM32F051R8 MCU on the STMF0Discovery board, compiled with Clang v3.5.0. We refer to the (publicly available) code for more detailed compiler settings. For both Diffie-Hellman and signatures we follow the eBACS [4] API.

\subsection{Core functionality}

The arithmetic of the underlying finite fields is well-studied and optimized, and we do not reinvent the wheel. For field arithmetic in $\mathbb{F}_{2^{255}-19}$ we use the highly optimized functions presented by Hutter and Schwabe [27] for the AVR ATmega, and the code from Düll et al. [17] for the Cortex M0. For arithmetic in $\mathbb{F}_{2^{127}-1}$ we use the functions from Renes et al. [39], which in turn rely on [27] for the AVR ATmega, and on [17] for the Cortex M0.

The SHAKE128 functions for the ATmega are taken from [10], while on the Cortex M0 we use a modified version from [2]. Cycle counts for the main functions defined in the rest of this paper are presented in Table 2. Notably, the Ladder routine is by far the most expensive function. In genus 1 the Compress function is relatively costly (it is essentially an inversion), while in genus 2 Check, Compress and Decompress have only minor impact on the total runtime. More interestingly, as seen in Table 3 and Table 4, the simplicity of operating only on the Kummer variety allows smaller code and less stack usage.

\footnotetext{
${ }^{9}$ The implementation decompresses $\pm R$ within Check, while Algorithm 3 assumes $\pm R$ to be decompressed. We have subtracted the cost of the Decompress function once.
} 


\begin{tabular}{|c|c|c|r|r|}
\hline Genus & Function & Ref. & AVR ATmega & ARM Cortex M0 \\
\hline \hline \multirow{4}{*}{1} & Ladder & Alg. 6 & 12539098 & 3338554 \\
& Check & Alg. 2 & 46546 & 17044 \\
& Compress & $\S 3.1$ & 1067004 & 270867 \\
& Decompress & $\S 3.1$ & 694 & 102 \\
\hline \hline \multirow{4}{*}{2} & Ladder & Alg. 9 & 9624637 & 2683371 \\
& Check & Alg. 3 & 84424 & 24249 \\
& Compress & Alg. 4 & 212374 & 62165 \\
& Decompress & Alg. 5 & 211428 & 62471 \\
\hline
\end{tabular}

Table 2. Cycle counts for the four key functions of qDSA at the 128-bit security level.

\subsection{Comparison to previous work}

There are not many implementations of complete signature and key exchange schemes on microcontrollers. On the other hand, there are implementations of scalar multiplication on elliptic curves. The current fastest on our platforms are presented by Düll et al. [17], and since we are relying on exactly the same arithmetic, we have essentially the same results. Similarly, the current records for scalar multiplication on Kummer surfaces are presented by Renes et al. [39]. Since we use the same underlying functions, we have similar results.

More interestingly, we compare the speed and memory usage of signing and verification to best known results of implementations of complete signature schemes. To the best of our knowledge, the only other works are the Ed25519-based scheme by Nascimento et al [34], the FourQQ-based scheme (obtaining fast scalar multiplication by relying on easily computable endomorphisms) by Liu et al [30], and the genus-2 implementation from [39].

AVR ATmega. As we see in Table 3, our implementation of the scheme based on Curve25519 outperforms the Ed25519-based scheme from [34] in every way. It reduces the number of clock cycles needed for sign resp. verify by more than $26 \%$ resp. $17 \%$, while reducing stack usage by more than $65 \%$ resp. $47 \%$. Code size is not reported in [34]. Comparing against the Four $\mathbb{Q}$ implementation of [30], we see a clear trade-off between speed and size: Four $\mathbb{Q}$ has a clear speed advantage, but qDSA on Curve25519 requires only a fraction of the stack space.

The implementation based on the Kummer surface of the genus-2 Gaudry-Schost Jacobian does better than the Curve25519-based implementation across the board. Compared to [39], the stack usage of sign resp. verify decreases by more than $54 \%$ resp. $38 \%$, while decreasing code size by about $11 \%$. On the other hand, verification is about $26 \%$ slower. This is explained by the fact that in [39] the signature is compressed to 48 bytes (following Schnorr's suggestion), which means that one of the scalar multiplications in verification is only half length. Comparing to the Four $\mathbb{Q}$ implementation of [30], again we see a clear trade-off between speed and size, but this time the loss of speed is less pronounced than in the comparison with Curve25519-based qDSA.

ARM Cortex M0. In this case there is no elliptic-curve-based signature scheme to compare to, so we present the first. As we see in Table 4, it is significantly slower than its genus- 2

$\overline{{ }^{10} \text { All reported }}$ code sizes except those from [30, Table 6] include support for both signatures and key exchange. 


\begin{tabular}{|c|c|c|r|r|r|}
\hline Ref. & Object & Function & Clock cycles & Stack & Code size $^{10}$ \\
\hline \hline \multirow{2}{*}[34]{} & \multirow{2}{*}{ Ed25519 } & sign & 19047706 & 1473 bytes & \\
& & verify & 30776942 & 1226 bytes & \\
\hline \multirow{2}{*}[30]{} & \multirow{2}{*}{ FourQQ } & sign & 5174800 & 1572 bytes & 25354 bytes \\
& & verify & 11003800 & 4957 bytes & 33372 bytes \\
\hline \multirow{2}{*}{ This work } & Curve25519 & sign & 14067995 & 512 bytes & \multirow{2}{*}{21347 bytes } \\
& & verify & 25355140 & 644 bytes & \\
\hline \hline \multirow{2}{*}[39]{} & Gaudry- & sign & 10404033 & 926 bytes & \multirow{2}{*}{20242 bytes } \\
& Schost $\mathcal{J}$ & verify & 16240510 & 992 bytes & \\
\hline \multirow{2}{*}{ This work } & Gaudry- & sign & 10477347 & 417 bytes & \multirow{2}{*}{17880 bytes } \\
& Schost $\mathcal{K}$ & verify & 20423937 & 609 bytes & \\
\hline
\end{tabular}

Table 3. Performance comparison of the qDSA signature scheme against the current best implementations, on the AVR ATmega platform.

counterpart in this paper (as should be expected), while using a similar amount of stack and code. The genus- 2 signature scheme has similar trade-offs on this platform when compared to the implementation by Renes et al. [39]. The stack usage for sign resp. verify is reduced by about $57 \%$ resp. $43 \%$, while code size is reduced by about $8 \%$. For the same reasons as above, verification is about $28 \%$ slower.

\begin{tabular}{|c|c|c|r|r|r|}
\hline Ref. & Object & Function & Clock cycles & Stack & Code size $^{11}$ \\
\hline \hline \multirow{2}{*}{ This work } & Curve25519 & $\begin{array}{c}\text { sign } \\
\text { verify }\end{array}$ & $\begin{array}{r}3889116 \\
6793695\end{array}$ & $\begin{array}{r}660 \text { bytes } \\
788 \text { bytes }\end{array}$ & 18443 bytes \\
\hline \hline \multirow{2}{*}[39]{} & Gaudry- & sign & 2865351 & 1360 bytes & 19606 bytes \\
& Schost $\mathcal{J}$ & verify & 4453978 & 1432 bytes & \\
\hline \multirow{2}{*}{ This work } & Gaudry- & sign & 2908215 & 580 bytes & \multirow{2}{*}{18064 bytes } \\
& Schost $\mathcal{K}$ & verify & 5694414 & 808 bytes & \\
\hline
\end{tabular}

Table 4. Performance comparison of the qDSA signature scheme against the current best implementations, on the ARM Cortex M0 platform.

Acknowledgements. We thank Peter Schwabe for his valuable contributions to discussions during the creation of this paper, and the anonymous Asiacrypt reviewers for their helpful comments.

\section{References}

1. Accredited Standards Committee X9. American National Standard X9.62-1999, Public key cryptography

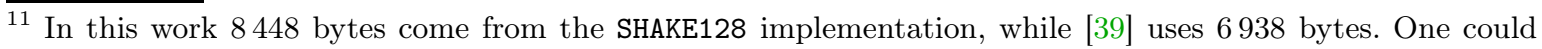
probably reduce this significantly by optimizing the implementation, or by using a more memory-friendly hash function. 
for the financial services industry: the elliptic curve digital signature algorithm (ECDSA). Technical report, ANSI, 1999. Preliminary draft at http://grouper.ieee.org/groups/1363/Research/Other.html. 1

2. E. Alkim, P. Jakubeit, and P. Schwabe. NewHope on ARM Cortex-M. In Security, Privacy, and Applied Cryptography Engineering - 6th International Conference, SPACE 2016, Hyderabad, India, December 1418, 2016, Proceedings, pages 332-349, 2016. 24

3. W. L. Baily, Jr. On the theory of $\theta$-functions, the moduli of abelian varieties, and the moduli of curves. Ann. of Math. (2), 75:342-381, 1962. 15

4. D. Bernstein and T. Lange. eBACS: ECRYPT Benchmarking of Cryptographic Systems. https://bench. cr.yp.to/index.html. Accessed: 2017-05-18. 24

5. D. J. Bernstein. Curve25519: new Diffie-Hellman speed records. In M. Yung, Y. Dodis, A. Kiayias, and T. Malkin, editors, Public Key Cryptography - PKC 2006, volume 3958 of Lecture Notes in Computer Science, pages 207-228. Springer-Verlag Berlin Heidelberg, 2006. http://cr.yp.to/papers.html\# curve25519. 1, 3, 10

6. D. J. Bernstein. Elliptic vs. hyperelliptic, part 1, 2006. http://cr.yp.to/talks/2006.09.20/slides . pdf. 2, 12, 18, 19

7. D. J. Bernstein, C. Chuengsatiansup, T. Lange, and P. Schwabe. Kummer strikes back: New DH speed records. In P. Sarkar and T. Iwata, editors, Advances in Cryptology - ASIACRYPT 2014, volume 8873 of LNCS, pages 317-337. Springer, 2014. https://cryptojedi.org/papers/\#kummer. 10, 12, 18

8. D. J. Bernstein, N. Duif, T. Lange, P. Schwabe, and B.-Y. Yang. High-speed high-security signatures. J. Cryptographic Engineering, 2(2):77-89, 2012. https://cryptojedi.org/papers/\#ed25519. 1, 2, 6, 8

9. D. J. Bernstein, T. Lange, and P. Schwabe. The security impact of a new cryptographic library. In Progress in Cryptology - LATINCRYPT 2012 - 2nd International Conference on Cryptology and Information Security in Latin America, Santiago, Chile, October 7-10, 2012. Proceedings, pages 159-176, 2012. 1

10. G. Bertoni, J. Daemen, M. Peeters, and G. V. Assche. The KecCAK sponge function family, 2016. http:// keccak.noekeon.org/. 24

11. J. W. Bos, C. Costello, H. Hisil, and K. E. Lauter. Fast cryptography in genus 2. In T. Johansson and P. Q. Nguyen, editors, Advances in Cryptology - EUROCRYPT 2013, volume 7881 of LNCS, pages 194-210. Springer, 2013. https://eprint.iacr.org/2012/670.pdf. 12

12. J. W. S. Cassels and E. V. Flynn. Prolegomena to a middlebrow arithmetic of curves of genus 2, volume 230. Cambridge University Press, 1996. 19

13. D. V. Chudnovsky and G. V. Chudnovsky. Sequences of numbers generated by addition in formal groups and new primality and factorization tests. Adv. in Appl. Math., 7:385-434, 1986. 12

14. P.-N. Chung, C. Costello, and B. Smith. Fast, uniform, and compact scalar multiplication for elliptic curves and genus 2 jacobians with applications to signature schemes. Cryptology ePrint Archive, Report 2015/983, 2015. https://eprint.iacr.org/2015/983. 2, 4

15. R. Cosset. Applications des fonctions theta à la cryptographie sur les courbes hyperelliptiques. PhD thesis, Université Henri Poincaré - Nancy I, 2011. https://tel.archives-ouvertes.fr/tel-00642951/file/ main.pdf. 12

16. W. Diffie and M. E. Hellman. New directions in cryptography. Information Theory, IEEE Transactions on, 22(6):644-654, 1976. 1

17. M. Düll, B. Haase, G. Hinterwälder, M. Hutter, C. Paar, A. H. Sánchez, and P. Schwabe. High-speed Curve25519 on 8-bit, 16-bit and 32-bit microcontrollers. Design, Codes and Cryptography, 77(2), 2015. http://cryptojedi.org/papers/\#mu25519. 8, 24, 25

18. M. J. Dworkin. SHA-3 standard: Permutation-based hash and extendable-output functions. Technical report, National Institute of Standards and Technology (NIST), 2015. http://www.nist.gov/ manuscript-publication-search. cfm?pub_id=919061. 6

19. A. Fiat and A. Shamir. How to prove yourself: Practical solutions to identification and signature problems. In Advances in Cryptology - CRYPTO '86, Santa Barbara, California, USA, 1986, Proceedings, pages 186194, 1986. 2, 5

20. T. E. Gamal. A Public Key Cryptosystem and a Signature Scheme Based on Discrete Logarithms. In Advances in Cryptology, Proceedings of CRYPTO '84, Santa Barbara, California, USA, August 19-22, 1984, Proceedings, pages 10-18, 1984. 8

21. P. Gaudry. Fast genus 2 arithmetic based on Theta functions. J. Mathematical Cryptology, 1(3):243-265, 2007. https://eprint.iacr.org/2005/314/. 1, 10, 12, 13, 14, 16

22. P. Gaudry and E. Schost. Genus 2 point counting over prime fields. J. Symb. Comput., 47(4):368-400, 2012. 3, 10, 17 
23. M. Hamburg. Fast and compact elliptic-curve cryptography. Cryptology ePrint Archive, Report 2012/309, 2012. http://eprint.iacr.org/2012/309. 2

24. M. Hamburg. The STROBE protocol framework. Cryptology ePrint Archive, Report 2017/003, 2017. http://eprint.iacr.org/2017/003. 2, 4

25. C. Hazay and Y. Lindell. Efficient Secure Two-Party Protocols. Springer-Verlag Berlin Heidelberg, 2010. 5

26. R. W. H. T. Hudson. Kummer's quartic surface. Cambridge University Press, 1905. 20

27. M. Hutter and P. Schwabe. $\mathrm{NaCl}$ on 8-bit AVR microcontrollers. In A. Youssef and A. Nitaj, editors, Progress in Cryptology - AFRICACRYPT 2013, volume 7918 of LNCS, pages 156-172. Springer, 2013. http://cryptojedi.org/papers/\#avrnacl. 24

28. S. Karati and A. Das. Faster batch verification of standard ECDSA signatures using summation polynomials. In I. Boureanu, P. Owesarski, and S. Vaudenay, editors, Applied Cryptography and Network Security: 12th International Conference, ACNS 2014, Lausanne, Switzerland, June 10-13, 2014. Proceedings, pages 438-456, Cham, 2014. Springer International Publishing. 2

29. N. Koblitz. Elliptic curve cryptosystems. Mathematics of Computation, 48:203-209, 1987. 1

30. Z. Liu, P. Longa, G. Pereira, O. Reparaz, and H. Seo. Four $\mathbb{Q}$ on embedded devices with strong countermeasures against side-channel attacks. Cryptology ePrint Archive, Report 2017/434, 2017. http:// eprint.iacr.org/2017/434. 25, 26

31. V. Miller. Use of Elliptic Curves in Cryptography. In Advances in Cryptology - CRYPTO 85 Proceedings, volume 218 of Lecture Notes in Computer Science, pages 417-426. Springer Berlin / Heidelberg, Berlin, Germany, 1986. 1

32. P. L. Montgomery. Speeding the Pollard and elliptic curve methods of factorization. Mathematics of Computation, 1987. 1, 3, 8

33. D. Naccache, D. M'Raïhi, S. Vaudenay, and D. Raphaeli. Can D.S.A. be improved? complexity trade-offs with the digital signature standard. In Advances in Cryptology - EUROCRYPT '94, Workshop on the Theory and Application of Cryptographic Techniques, Perugia, Italy, May 9-12, 1994, Proceedings, pages $77-85,1994.8$

34. E. Nascimento, J. López, and R. Dahab. Efficient and secure elliptic curve cryptography for 8-bit avr microcontrollers. In R. S. Chakraborty, P. Schwabe, and J. Solworth, editors, Security, Privacy, and Applied Cryptography Engineering, volume 9354 of LNCS, pages 289-309. Springer, 2015. 25, 26

35. K. Okeya and K. Sakurai. Efficient elliptic curve cryptosystems from a scalar multiplication algorithm with recovery of the y-coordinate on a Montgomery-form elliptic curve. In Ç. K. Koç, D. Naccache, and C. Paar, editors, Cryptographic Hardware and Embedded Systems - CHES 2001, volume 2162 of LNCS, pages 126-141. Springer, 2001. 2, 4

36. T. Perrin. The XEdDSA and VXEdDSA Signature Schemes. https://whispersystems.org/docs/ specifications/xeddsa/. Accessed: 2017-05-18. 2

37. D. Pointcheval and J. Stern. Security proofs for signature schemes. In Advances in Cryptology - EUROCRYPT '96, International Conference on the Theory and Application of Cryptographic Techniques, Saragossa, Spain, May 12-16, 1996, Proceeding, pages 387-398, 1996. 2, 5, 6

38. D. Pointcheval and J. Stern. Security arguments for digital signatures and blind signatures. J. Cryptology, 13(3):361-396, 2000. 2, 5, 6

39. J. Renes, P. Schwabe, B. Smith, and L. Batina. $\mu$ Kummer: Efficient hyperelliptic signatures and key exchange on microcontrollers. In Cryptographic Hardware and Embedded Systems - CHES 2016 - 18 th International Conference, Santa Barbara, CA, USA, August 17-19, 2016, Proceedings, pages 301-320, 2016. 10, 12, 18, 24, 25, 26, 30

40. C.-P. Schnorr. Efficient identification and signatures for smart cards. In G. Brassard, editor, Advances in Cryptology - CRYPTO '89, volume 435 of $L N C S$, pages 239-252. Springer, 1989. 1, 2, 4, 8

41. I. A. Semaev. Summation polynomials and the discrete logarithm problem on elliptic curves. IACR Cryptology ePrint Archive, 2004:31, 2004. 2

42. C. Stahlke. Point compression on jacobians of hyperelliptic curves over $\mathbb{F}_{q}$. Cryptology ePrint Archive, Report 2004/030, 2004. https://eprint.iacr.org/2004/030. 19

\section{A Elliptic implementation details}

The algorithms in this section complete the description of elliptic qDSA in $\S 3$. 


\section{A.1 Pseudoscalar multiplication}

The keypair, sign, and verify functions all require Ladder, which we define below. Algorithm 6 describes the scalar pseudomultiplication that we implemented for Montgomery curves, closely following our $\mathrm{C}$ reference implementation. To make our Ladder constant-time, we use a conditional swap procedure CSWAP. This takes a single bit and a pair of items as arguments, and swaps those items if and only if the bit is 1 .

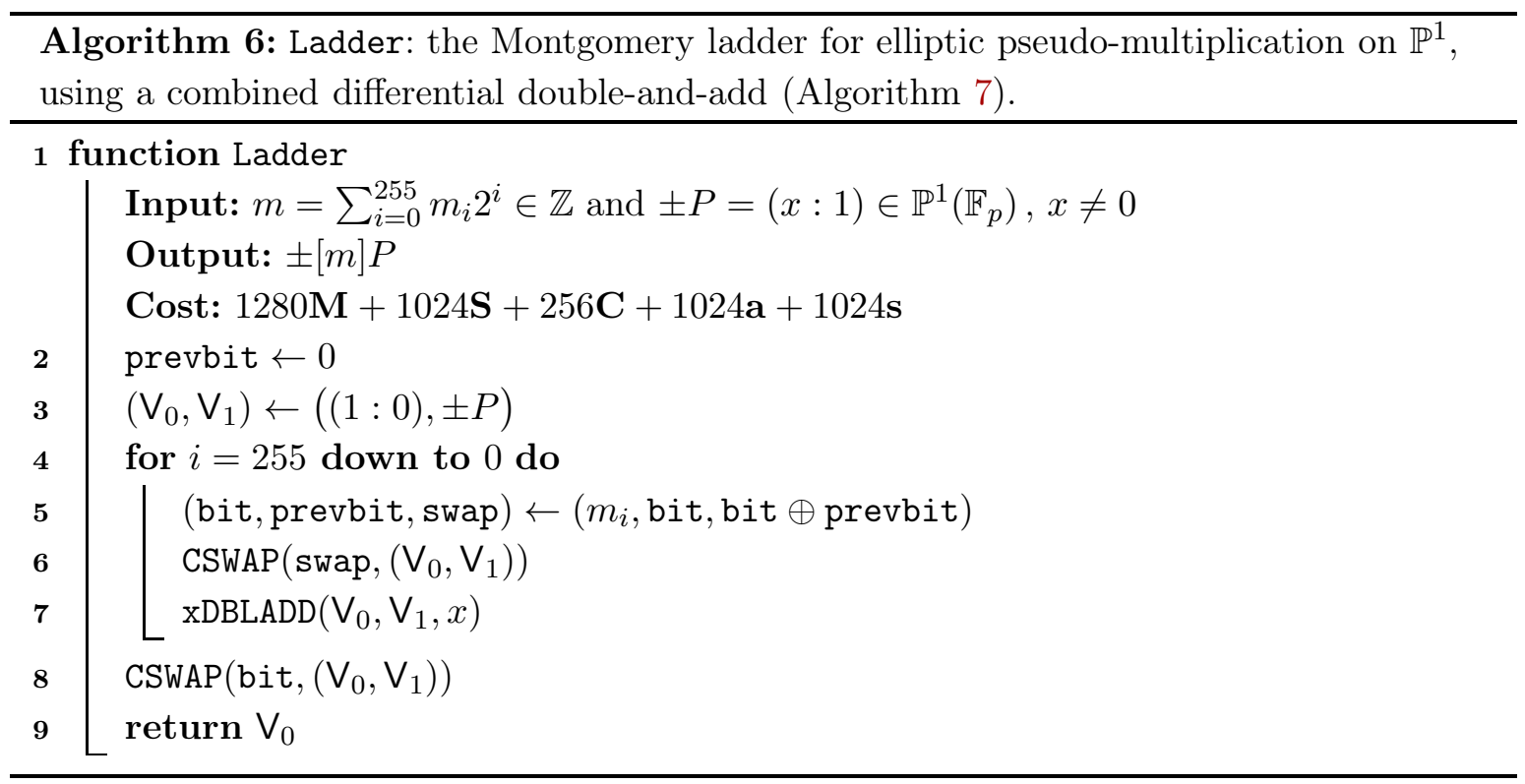

Algorithm 7 implements xDBLADD for Montgomery curves in the usual way. Note that the assumption that $\pm(P-Q) \notin\{(1: 0),(0: 1)\}$ implies that xDBLADD will always return the correct result.

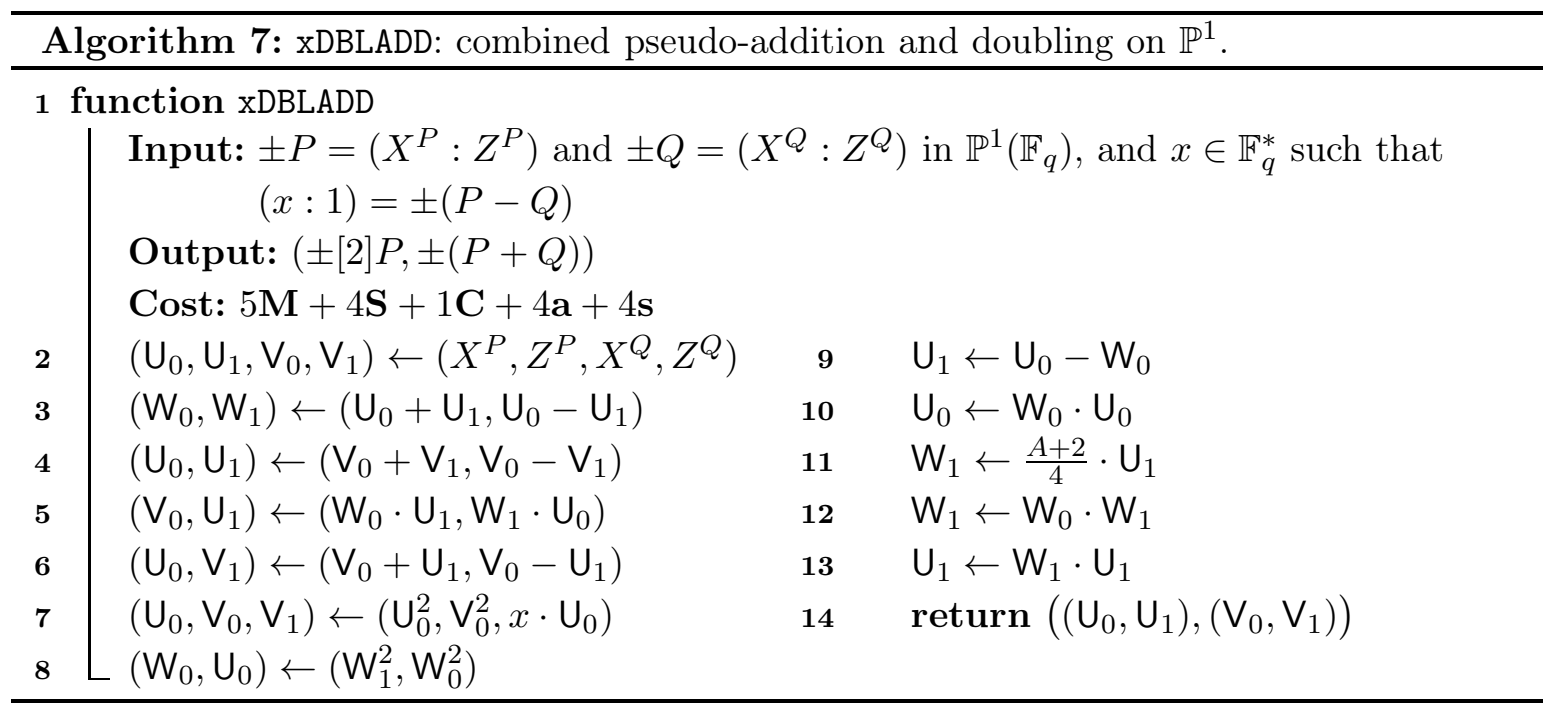




\section{A.2 The BValues subroutine for signature verification}

The elliptic version of the crucial Check subroutine of verify (Algorithm 2) used a function BValues to calculate the values of the biquadratic forms $B_{X X}, B_{X Z}$, and $B_{Z Z}$. This function can be implemented in a number of ways, with different optimizations for speed or stack usage. Algorithm 8 illustrates the approach we used for BValues, motivated by simplicity and stack minimisation.

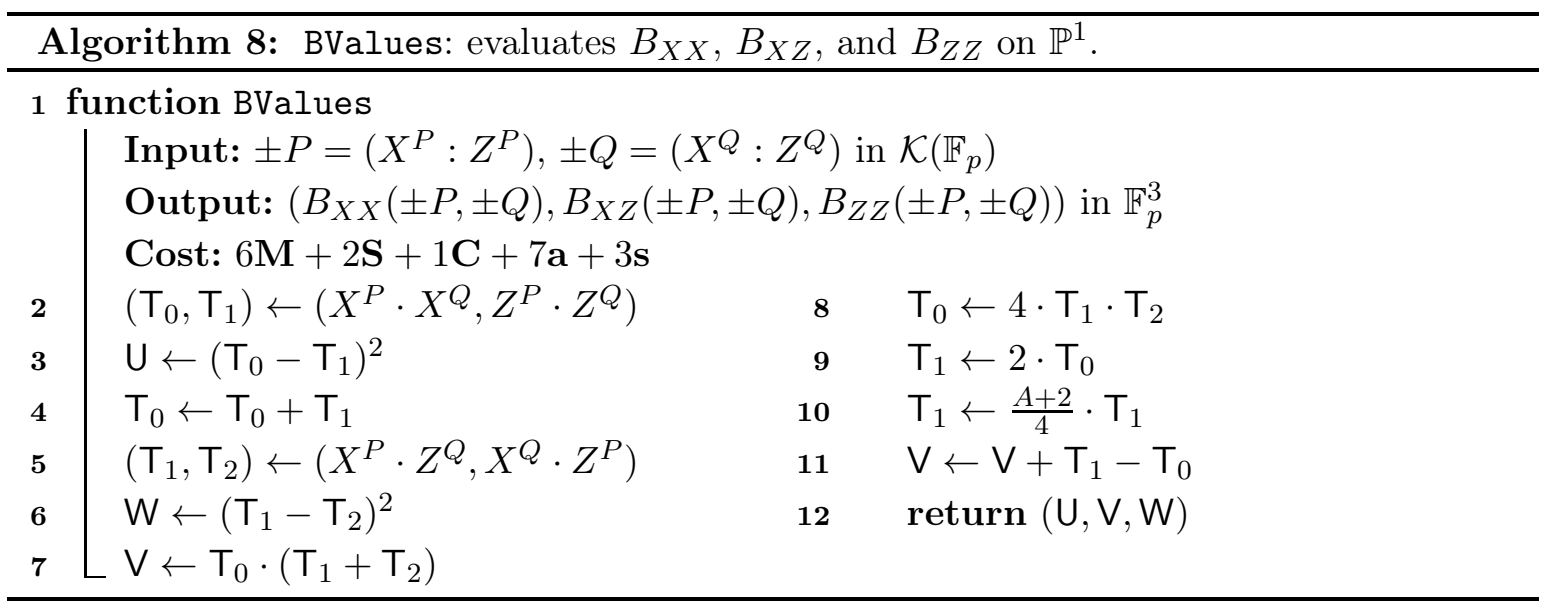

\section{B Kummer surface implementation details}

The algorithms in this section complete the description of Kummer qDSA in $\S \S 4-6$. They follow our $\mathrm{C}$ reference implementation very closely. Recall that we have the following subroutines:

- Mul4 implements a 4-way parallel multiplication. It takes a pair of vectors $\left(x_{1}, x_{2}, x_{3}, x_{4}\right)$ and $\left(y_{1}, y_{2}, y_{3}, y_{4}\right)$ in $\mathbb{F}_{p}^{4}$, and returns $\left(x_{1} y_{1}, x_{2} y_{2}, x_{3} y_{3}, x_{4} y_{4}\right)$.

- Sqr4 implements a 4 -way parallel squaring. Given a vector $\left(x_{1}, x_{2}, x_{3}, x_{4}\right)$ in $\mathbb{F}_{p}^{4}$, it returns $\left(x_{1}^{2}, x_{2}^{2}, x_{3}^{2}, x_{4}^{2}\right)$.

- Had implements a Hadamard transform. Given a vector $\left(x_{1}, x_{2}, x_{3}, x_{4}\right)$ in $\mathbb{F}_{p}^{4}$, it returns $\left(x_{1}+x_{2}+x_{3}+x_{4}, x_{1}+x_{2}-x_{3}-x_{4}, x_{1}-x_{2}+x_{3}-x_{4}, x_{1}-x_{2}-x_{3}+x_{4}\right)$.

- Dot computes the sum of a 4-way multiplication. Given a pair of vectors $\left(x_{1}, x_{2}, x_{3}, x_{4}\right)$ and $\left(y_{1}, y_{2}, y_{3}, y_{4}\right)$ in $\mathbb{F}_{p}^{4}$, it returns $x_{1} y_{1}+x_{2} y_{2}+x_{3} y_{3}+x_{4} y_{4}$.

\section{B.1 Scalar pseudomultiplication}

The Montgomery Ladder for scalar pseudomultiplication on $\mathcal{K}^{\mathrm{Sqr}}$ is implemented in Algorithm 9, replicating the approach in [39]. It relies on the WRAP and xDBLADD functions, implemented in Algorithm 10 respectively 11. The function WRAP takes a Kummer point $\pm P$ in $\mathcal{K}^{\operatorname{Sqr}}\left(\mathbb{F}_{p}\right)$ and returns $w_{2}, w_{3}$, and $w_{4}$ in $\mathbb{F}_{p}$ such that $\left(1: w_{2}: w_{3}: w_{4}\right)=\left(1 / X_{1}^{P}: 1 / X_{2}^{P}:\right.$ $\left.1 / X_{3}^{P}: 1 / X_{4}^{P}\right)$. The resulting values are required in every xDBLADD within Ladder; the idea is to compute them once with a single inversion at the start of the procedure, thus avoiding further expensive inversions. We note that this "wrapped" form of the point $\pm P$ was previously used as a compressed form for Kummer point transmission, but since it requires three full field values it is far from an optimal compression. 

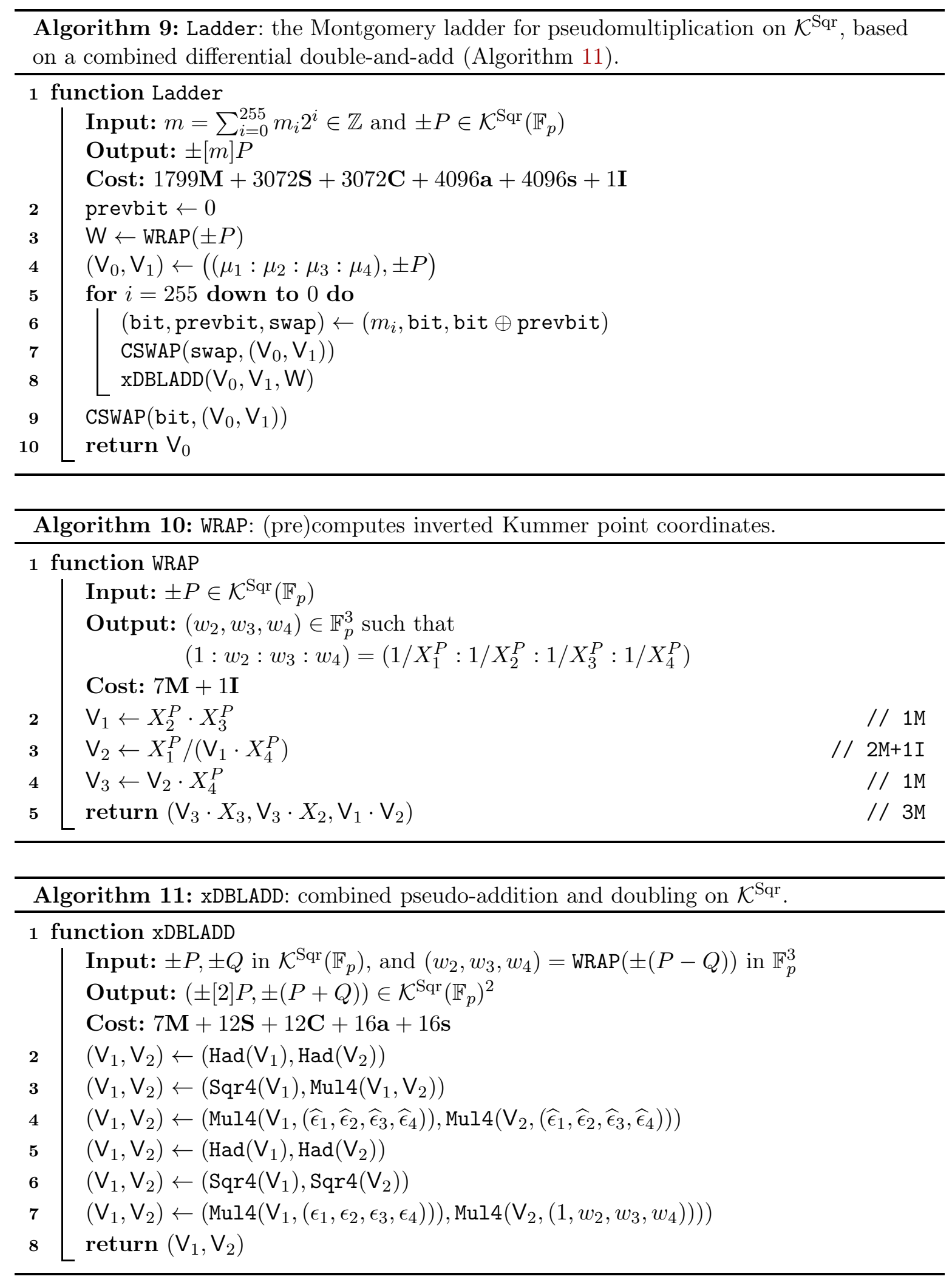


\section{B.2 Subroutines for signature verification}

The crucial Check function for $\mathcal{K}^{\mathrm{Sqr}}$ (Algorithm 3) calls subroutines BiiValues and BijValue to compute the values of the biquadratic forms on $\mathcal{K}^{\text {Int }}$. Algorithms 12 and 13 are our simple implementations of these functions. We choose to only store the four constants $\widehat{\mu}_{1}, \widehat{\mu}_{2}, \widehat{\mu}_{3}$ and $\widehat{\mu}_{4}$, but clearly one can gain some efficiency by pre-computing more constants (eg. $\widehat{\mu}_{1} \widehat{\mu}_{2}$, $\widehat{\mu}_{1} \widehat{\mu}_{4}-\widehat{\mu}_{2} \widehat{\mu}_{3}$, etc.). As the speed of this operation is not critical, it allows us to reduce the number of necessary constants. The four values of $B_{11}, B_{22}, B_{33}$, and $B_{44}$ are computed simultaneously, since many of the intermediate operands are shared (as is clear from Equations (16) through (19)).

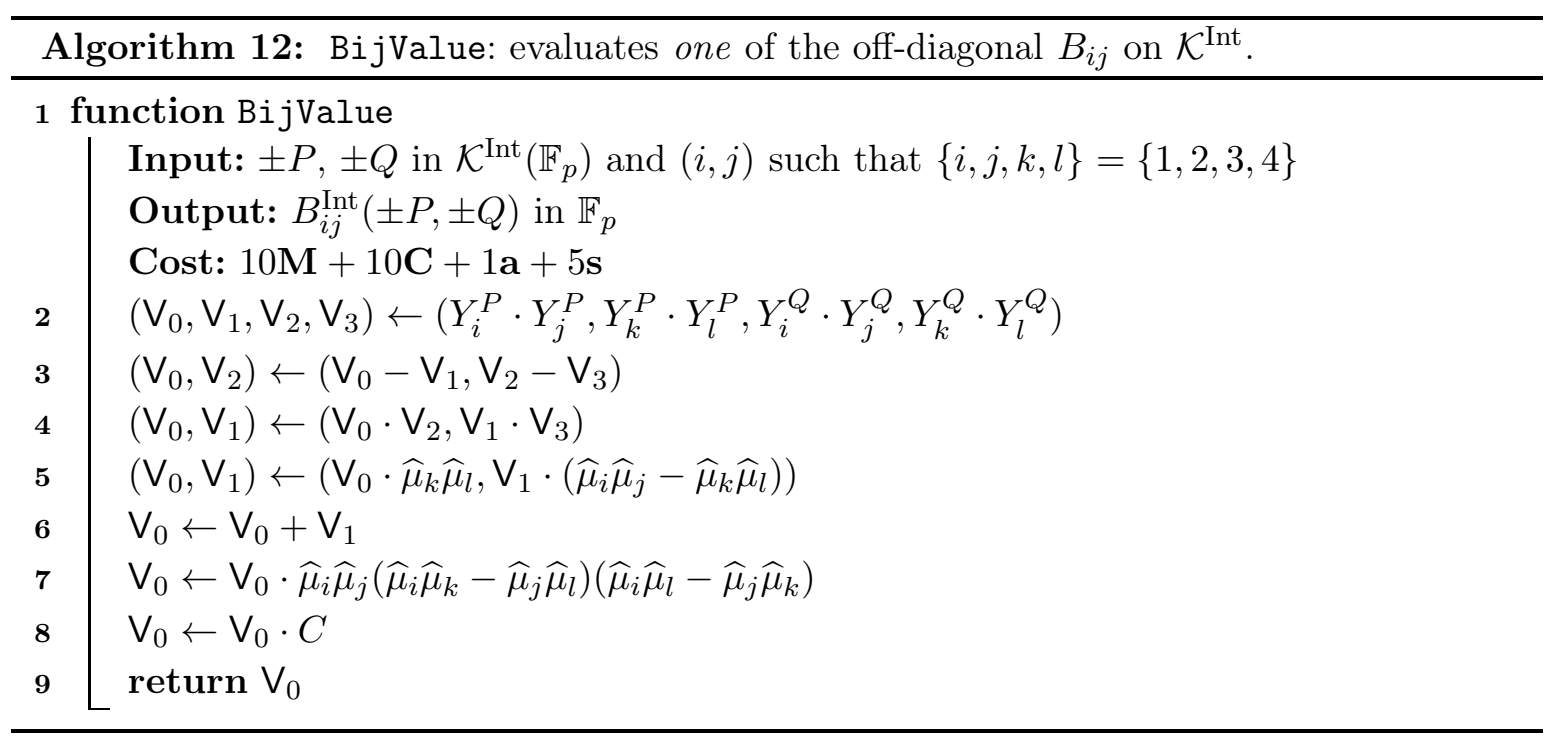

\section{B.3 Subroutines for compression and decompression}

The compression and decompression functions in Algorithms 4 and 5 require the evaluation of the polynomials $K_{2}, K_{3}$, and $K_{4}$. We used the simple strategy in Algorithms 14, 15, and 16 (get_ $K_{2}$, get_ $K_{3}$, and get_ $K_{4}$, respectively), which prioritises low stack usage over speed (which is again not critical here). 

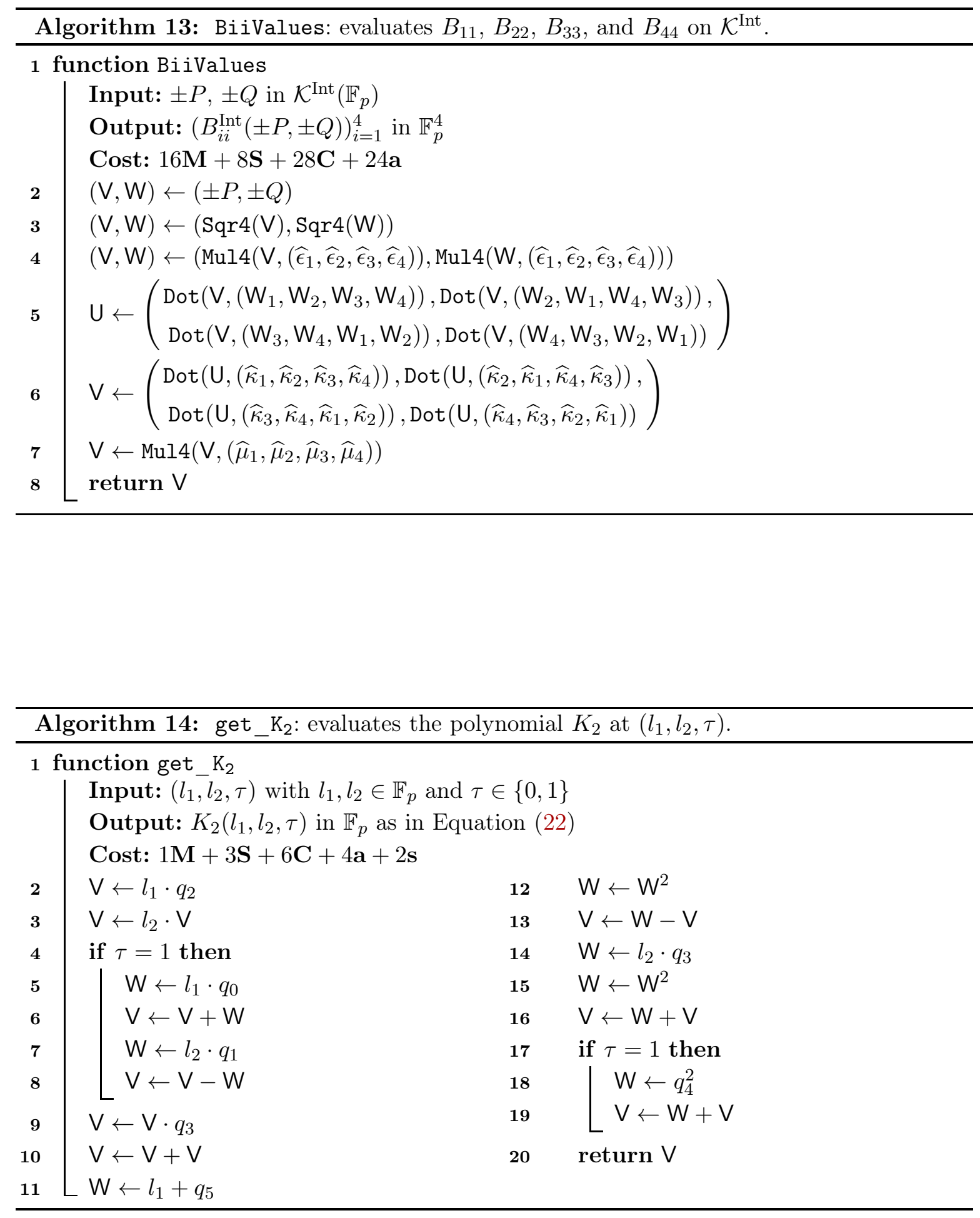


\begin{tabular}{|c|c|c|c|}
\hline \multicolumn{4}{|c|}{ Algorithm 15: get_ $\mathrm{K}_{3}$ : evaluates the polynomial $K_{3}$ at $\left(l_{1}, l_{2}, \tau\right)$. } \\
\hline \multicolumn{4}{|c|}{1 function get_ $K_{3}$} \\
\hline & \multicolumn{3}{|c|}{ Input: $\left(l_{1}, l_{2}, \tau\right)$ with $l_{1}, l_{2} \in \mathbb{F}_{p}$ and $\tau \in\{0,1\}$} \\
\hline & \multicolumn{3}{|c|}{ Output: $K_{3}\left(l_{1}, l_{2}, \tau\right)$ in $\mathbb{F}_{p}$ as in Equation $(23)$} \\
\hline & \multicolumn{3}{|c|}{ Cost: $3 \mathbf{M}+2 \mathbf{S}+6 \mathbf{C}+4 \mathbf{a}+2 \mathbf{s}$} \\
\hline 2 & $\mathrm{U} \leftarrow l_{2}^{2}$ & 13 & $\mathrm{~V} \leftarrow \mathrm{V}-\mathrm{U}$ \\
\hline 3 & $\mathrm{~V} \leftarrow l_{1}^{2}$ & 14 & if $\tau=1$ then \\
\hline 4 & if $\tau=1$ then & 15 & $\mathrm{~V} \leftarrow \mathrm{V}+\mathrm{W}$ \\
\hline 5 & $\mathrm{~W} \leftarrow \mathrm{U}+\mathrm{V}$ & 16 & $\mathrm{~V} \leftarrow \mathrm{V} \cdot q_{3}$ \\
\hline 6 & $\mathrm{~W} \leftarrow \mathrm{W} \cdot q_{2}$ & & if $\tau=1$ then \\
\hline 7 & $\mathrm{U} \leftarrow \mathrm{U}+1$ & 18 & $\mathrm{U} \leftarrow l_{1} \cdot l_{2}$ \\
\hline 8 & $\mathrm{~L} \leftarrow \leftarrow \mathrm{V}+1$ & 19 & $\mathrm{U} \leftarrow \mathrm{U} \cdot q_{6}$ \\
\hline 9 & $\mathrm{U} \leftarrow \mathrm{U} \cdot l_{1}$ & 20 & $\mathrm{U} \leftarrow \mathrm{U} \cdot q_{7}$ \\
\hline 10 & $\mathrm{~V} \leftarrow \mathrm{V} \cdot l_{2}$ & 21 & $V \leftarrow V-U$ \\
\hline 11 & $\mathrm{U} \leftarrow \mathrm{U} \cdot q_{1}$ & 22 & return V \\
\hline 12 & $\mathrm{~V} \leftarrow \mathrm{V} \cdot q_{0}$ & & \\
\hline
\end{tabular}

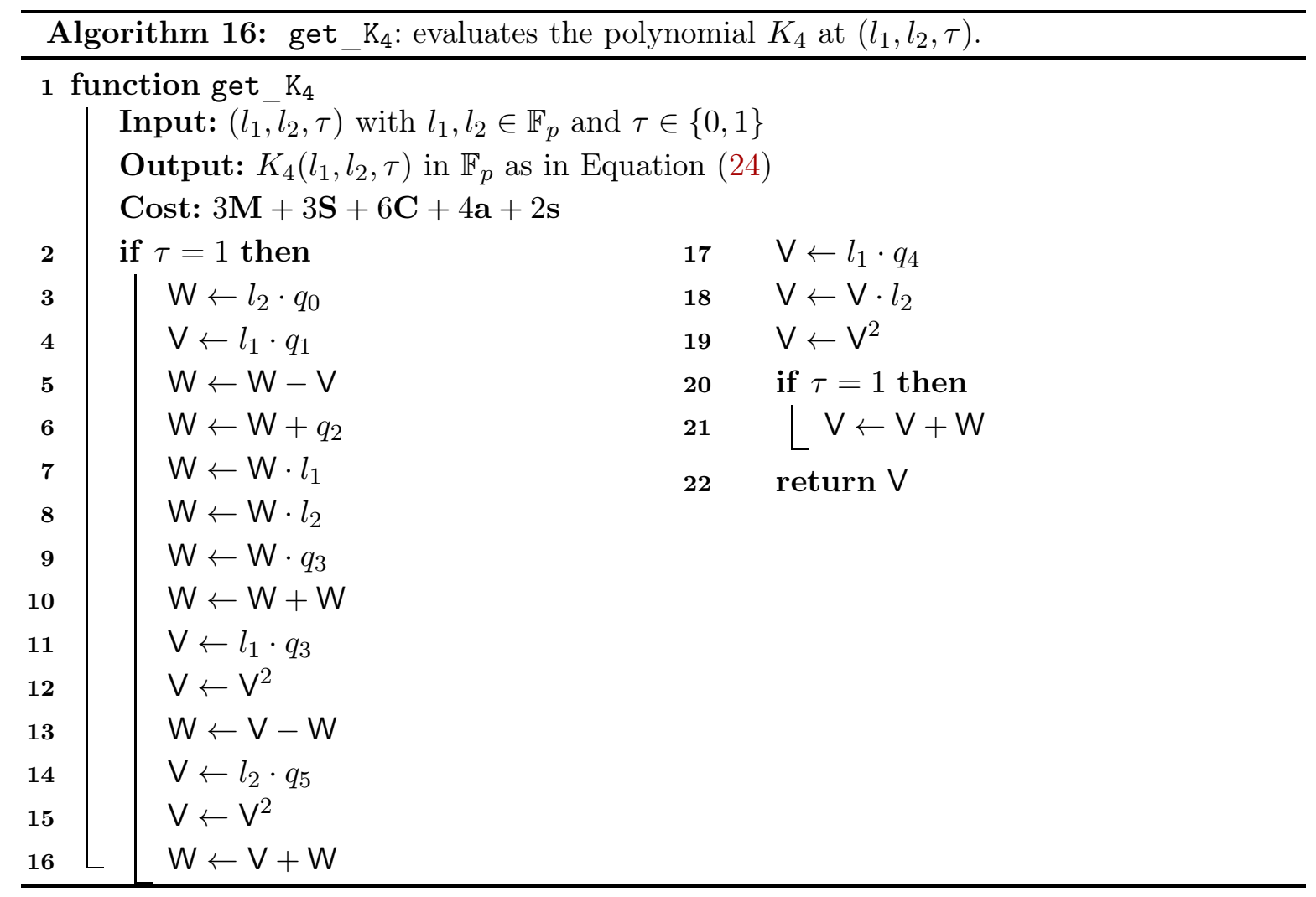

